\title{
Les trajectoires professionnelles en début de vie active : quel impact des contrats temporaires?
}

\author{
Sylvie Blasco* et Pauline Givord**
}

\begin{abstract}
L'impact de la flexibilité du marché du travail sur l'insertion des jeunes reste un objet de controverse. Si occuper un emploi temporaire permet d'acquérir de l'expérience professionnelle et peut constituer un « tremplin » vers un emploi stable, le risque existe de se retrouver dans une « voie de garage ». L'objet de cette étude est donc d'apporter des éléments de réponses empiriques à ces questions, en s'interrogeant sur les transitions entre emploi temporaire, emploi stable et non-emploi des personnes en début de vie active. Nous utilisons pour cela l'enquête Formation et Qualification Professionnelle de 2003 dont le calendrier fournit une description précise des trajectoires des personnes interrogées sur cinq années. Une modélisation "multi-états multi-épisodes » permet d'isoler et de mesurer l'impact respectif des caractéristiques individuelles observées, de l'hétérogénéité inobservée, des dépendances d'états passées et actuelles (le fait d'occuper tel ou tel emploi peut avoir une conséquence sur les trajectoires ultérieures) et des dépendances de durées (le fait de rester longtemps dans un état a des effets sur les chances d'en sortir) sur les processus d'insertion professionnelle. Les estimations mettent en évidence l'influence importante des caractéristiques individuelles sur les transitions. Elles montrent également que le fait de passer par tel ou tel état du marché du travail peut avoir un effet à long terme sur la trajectoire professionnelle. Passer par un emploi temporaire en début de carrière peut donc avoir une incidence sur le plus long terme. Plus précisément, l'emploi temporaire conduit plus souvent au non-emploi (chômage et inactivité) qu'à l'emploi stable. Cependant, les transitions vers l'emploi stable évoluent de manière non linéaire avec le temps passé dans un emploi temporaire. Les caractéristiques individuelles inobservées semblent peu importantes pour les hommes, mais le seraient davantage pour les femmes.
\end{abstract}


$\mathbf{L}$ e marché du travail français s'est flexibilisé à un rythme rapide depuis - au moins - le milieu des années 1980. Le recours aux contrats à durée déterminée (CDD) et à l'intérim, favorisé par des modifications successives du code du travail, est devenu aujourd'hui largement répandu. S'ils restent minoritaires dans l'emploi salarié, les emplois temporaires représentent désormais une part importante des embauches. En mai 2006, plus de deux tiers des embauches dans le secteur privé se sont faits sous contrat à durée déterminée (Lutinier, 2007). Par ailleurs, en réponse aux ralentissements économiques et aux difficultés à s'insérer sur le marché de l'emploi rencontrées par certaines catégories d'actifs, des contrats aidés à durée limitée (contrats emploi solidarité, contrats emplois consolidés, contrat initiative emploi...) ont été mis en place. $\mathrm{Au}$ cœur des dynamiques du marché du travail (Fondeur et Minni, 2006), les jeunes sont les premiers concernés par cette évolution de 1'emploi : en 2004, plus d'un jeune actif sur cinq occupe un emploi temporaire (1) (Givord, 2006).

Cette flexibilisation accrue du marché du travail est encore l'objet d'un débat passionné. Les emplois temporaires peuvent permettre à une main-d'œuvre peu expérimentée d'accéder à des premiers emplois, qui seraient ainsi des marchepieds vers des situations d'emploi stable. Dans un contexte où les signaux envoyés par le diplôme peuvent être brouillés, les entreprises hésiteraient devant une embauche définitive de jeunes sans expérience. L'emploi temporaire est de fait souvent utilisé comme une période d'essai prolongé, qui peut donc déboucher à terme sur une embauche définitive. Givord (2006) observe ainsi que près d'un tiers des salariés en CDD en mars 2001 étaient en contrat à durée indéterminée (CDI) dans la même entreprise l'année suivante.

A contrario, certains s'inquiètent de cette évolution qui fait peser l'essentiel de l'incertitude sur le salarié. L'accès à un emploi en CDI conditionne de nombreux aspects de l'insertion sociale, comme l'accès au logement. Le risque d'exposition au chômage fait craindre également une dualisation du marché du travail : les personnes les plus fragiles pourraient se retrouver durablement confinées dans des trajectoires « instables », alternant périodes de chômage et « petits boulots » sans avenir. Pour reprendre des termes fréquemment utilisés, ces emplois temporaires seraient plus des « trappes à précarité » que des « tremplins ».
Plusieurs raisons expliquent qu'il est difficile, voire impossible, d'apporter une réponse définitive à ce débat. La première tient à la difficulté à mesurer l'insertion. Celle-ci est constituée de multiples facettes qu'on ne peut appréhender par un indicateur unique. Le niveau de salaire, l'adéquation avec les qualifications obtenues ou à l'inverse le déclassement ressenti sont par exemple des aspects importants de la qualité de l'insertion. La « précarité » ne se mesure pas uniquement par la nature du contrat de travail, et l'accès à un emploi en contrat indéterminé n'est pas forcément une garantie suffisante d'insertion (Paugam, 2000).

Une deuxième limite est que, même en se restreignant à un aspect simple comme le contrat de travail, il n'existe pas de « mesure » objective permettant d'établir que les emplois temporaires freinent ou accélèrent l'accès à un emploi stable. Le fait que certains emplois temporaires débouchent sur un emploi stable, quand d'autres non, n'apporte en soi qu'une information limitée : la question pertinente est de déterminer ce qu'il en aurait été de l'insertion de ces personnes, si elles n'avaient pas occupé un emploi temporaire. Pour illustrer de manière caricaturale cette question, on peut penser au cas hypothétique d'une personne sans emploi qui recevrait une offre d'emploi temporaire : a-t-elle intérêt à accepter cette offre, ou au contraire à attendre une offre en CDI ? Cette reformulation de la question montre qu'il est illusoire d'attendre une mesure directe de l'impact des emplois temporaires sur l'insertion : au mieux peut-on avoir une mesure relative en prenant les chômeurs comme référence. Mais cette approche est évidemment compliquée par la présence d'effets de composition : dans l'exemple précédent, on peut supposer que les personnes qui choisiront in fine d'occuper un emploi temporaire ne sont pas les mêmes (en termes d'opportunités futures en particulier) que celles qui préféreront attendre une meilleure offre.

Une troisième raison tient à la complexité et la diversité des trajectoires professionnelles. Pour y répondre correctement, il est nécessaire d'observer des personnes sur une longue période, ce qui est rarement possible. Lorsque c'est le cas, l'analyse est complexe. Le simple fait d'occuper un emploi temporaire ou de passer par le

1. Dans la suite de l'article, les termes emploi temporaire et contrat court sont employés indifféremment pour désigner toute forme d'emploi à durée déterminée, soit les $C D D$, missions d'interim, et emplois aidés. 
chômage a sans doute un effet sur la trajectoire future, mais on peut également penser que c'est aussi la durée passée dans ce type d'emploi ou encore la récurrence de tels épisodes, qui a un impact. Cela signifie encore que les réponses apportées risquent de n'être pas univoques, et qu'il est nécessaire d'examiner l'effet des emplois temporaires sous ces différentes dimensions.

Cette étude apporte des éléments de réponse au débat sur les conséquences pour les jeunes du passage par un emploi temporaire. Nous utilisons ici l'enquête Formation et Qualification Professionnelle 2003 de l'Insee, en nous restreignant aux personnes entrées sur le marché du travail depuis moins de dix ans en 2003. Cette enquête fournit un calendrier détaillé des parcours professionnels sur cinq ans, et constitue donc une source privilégiée (et à notre connaissance, encore inexploitée) pour évaluer l'impact des emplois temporaires sur l'insertion des jeunes actifs. Compte tenu des remarques précédentes, il ne s'agit pas d'apporter une réponse définitive au débat, mais plus modestement de décrire les transitions éventuelles des emplois temporaires vers l'emploi stable, en tentant d'isoler les différentes dimensions décrites supra. Il s'agit donc de caractériser les déterminants des transitions professionnelles : vers quel type d'emploi les emplois temporaires conduisent-ils plus fréquemment? Quelle est l'incidence de la durée passée dans un tel emploi ? De la trajectoire professionnelle passée ? Comment les caractéristiques individuelles des personnes, observables ou non, influencent-elles ces transitions?

Le modèle dit " multi-états multi-épisodes » utilisé dans cette étude permet de mieux rendre compte de toute la dynamique des processus d'insertion. Plus précisément, il permet de distinguer ce qui relève de la " dépendance d'état » (le fait d'occuper tel ou tel emploi peut avoir une conséquence sur les trajectoires ultérieures), de la « dépendance de durée » (le fait de rester longtemps dans un état - le chômage par exemple - a des effets sur les chances d'en sortir), et de l'hétérogénéité individuelle (tous les actifs n'ont pas les mêmes chances d'obtenir tel ou tel emploi). Par le biais de variables explicatives décrivant le passé professionnel, il est possible de mesurer l'impact du passage par l'emploi précaire sur les transitions professionnelles, et d'évaluer ainsi la "dépendance d'état ».

\section{Chômage, emplois temporaires et insertion professionnelle : une revue de la littérature}

C onséquence de la flexibilisation des marchés du travail européens, les travaux empiriques sur les emplois temporaires ont été nombreux au cours de la dernière décennie. L'angle a porté sur le devenir des personnes occupant ce type d'emploi. Pour reprendre le titre de l'article de Booth et al. (2002), il s'agit de déterminer si ces emplois constituent des « tremplins » vers l'emploi stable ou des « trappes à précarité ». Cancé et Fréchou (2003) pour la France, McGinnity et al. (2005) pour l'Allemagne, proposent ainsi une description des devenirs professionnels des personnes ayant occupé de tels emplois. Pour utiles qu'elles soient, ces descriptions ne permettent pas de déterminer l'impact propre du fait d'occuper un emploi temporaire sur la trajectoire professionnelle. La question sous-jacente est de déterminer si une personne qui a occupé un tel emploi aurait connu la même trajectoire si elle ne l'avait pas occupé. Les difficultés « techniques » pour y répondre sont au moins de deux ordres.

La première tient aux effets probables de biais de composition. A priori, le fait qu'une personne occupe un emploi temporaire en début de vie active n'est pas (totalement) dû au hasard. Le diplôme, ou le genre peuvent, par exemple, créer de grandes différences dans les chances respectives d'occuper un emploi ou non en sortant du système scolaire. Ces caractéristiques jouent également un rôle sur le type de contrat qu'on peut se voir proposer. Elles sont aussi susceptibles d'influer sur la trajectoire future. Havet (2006) montre ainsi que les trajectoires professionnelles divergent fortement entre hommes et femmes, mais également que le passé professionnel n'a pas la même importance pour les hommes et pour les femmes. Cependant, s'il est possible de prendre en compte un certain nombre de ces déterminants joints sur la probabilité d'occuper un emploi temporaire et sur la carrière future, toutes ces caractéristiques ne sont pas observables. Il en est ainsi de la motivation ou de l'attachement plus ou moins fort au marché du travail ; de l'appartenance à des réseaux familiaux ou professionnels ; enfin de compétences professionnelles plus difficilement objectivables et donc mesurables.

Pour s'affranchir de ces effets de sélection liés à ces déterminants inobservables, Autor et 
Houseman (2005) utilisent une expérience intervenue dans l'état américain du Michigan, où les bénéficiaires de l'aide sociale étaient affectés aléatoirement à des opérateurs privés de placement. Ces opérateurs ont des recours différents à 1 '« emploi temporaire » (en fait l'équivalent du travail intérimaire dans le cadre français) : les bénéficiaires ont donc une probabilité différente de se voir proposer (et donc d'occuper) un emploi intérimaire selon l'opérateur auquel ils ont été confiés. Une partie de cette variation est indépendante des caractéristiques propres des personnes concernées, puisqu'elle dépend de l'affectation aléatoire à tel ou tel opérateur. Autor et Houseman (2005) montrent que dans le cadre américain les emplois temporaires ne favorisent pas l'insertion à long terme des publics en difficulté.

Ni les résultats ni la méthode de cette étude ne sont généralisables. Dans le cas du marché $\mathrm{du}$ travail américain où domine la doctrine de l' « employment at will », c'est-à-dire l'embauche sans contrat de travail (2), l'usage de l'emploi temporaire est marginal. Il s'adresse potentiellement à un public très spécifique, par exemple d'anciennes personnes incarcérées ou travailleurs clandestins (Chauvin, 2010). Par ailleurs, si l'affectation aléatoire est la solution la plus rigoureuse pour réduire les problèmes de sélection, elle est évidemment très rare et difficilement utilisable en général. Dans le cadre français et plus largement européen, les différents contrats peuvent être utilisés par l'ensemble des entreprises et proposés à l'ensemble des salariés ; il est donc difficile d'imaginer un cadre crédible pour une telle expérience aléatoire.

À défaut, Ichino et al. (2008) s'appuient sur une hypothèse plus forte, l'indépendance conditionnelle aux variables observables. Elle suppose que les données contiennent suffisamment d'informations sur les personnes pour prendre en compte les effets de sélection. Plus précisément, l'autorisation du recours à l'intérim étant assez récente en Italie, ils supposent que pour des personnes ayant des profils très proches (en particulier en termes d'histoire professionnelle passée et surtout de distance à l'agence de travail temporaire la plus proche), le fait de s'adresser ou pas à une agence de travail temporaire n'est pas lié à des effets de sélection. Les auteurs trouvent ainsi un impact positif de l'intérim sur la probabilité d'accès à l'emploi stable. Ces résultats vont à l'encontre de ceux de Autor et Houseman (2005), ce qui est justifié par les différences institutionnelles importantes existant entre les États-Unis et l'Italie. Il est à noter cependant que l'hypothèse d'indépendance conditionnelle mobilisée par Ichino et al. (2008) est évidemment forte et contestable. En général, les études tentent donc de mieux prendre en compte la présence d'hétérogénéité inobservée.

La seconde difficulté à laquelle se heurte cette évaluation tient à la nature des processus étudiés. Elle suppose en effet d'observer des trajectoires professionnelles sur une longue période. Ces trajectoires sont multiples : les personnes peuvent occuper différents états sur le marché du travail, et ce pendant des durées variables. S'il est impossible de décrire toutes les trajectoires, on peut tenter d'en dresser une typologie ; c'est ce que fait par exemple Lopez (2004) pour décrire les différents modes d'insertion des sortants du système scolaire de l'enquête Génération 1998. Pour répondre à la question plus précise de l'impact causal des contrats courts sur l'accès à l'emploi stable, les études s'intéressent en général aux transitions relatives entre emploi court et emploi stable d'une part, et chômage et emploi stable de l'autre. En termes techniques, il s'agit de distinguer la « dépendance d'état », c'est-à-dire l'incidence d'occuper tel ou tel état du marché du travail, de l'hétérogénéité inobservée sur la trajectoire future.

La première approche statistique classique dans la littérature empirique consiste à modéliser, à partir de données discrètes, les transitions entre les différents états, en utilisant des modèles logistiques polytomiques dynamiques. L'avantage de cette méthode est un traitement assez satisfaisant de l'hétérogénéité inobservée. Dans Magnac (2000), elle est ainsi prise en compte par des effets fixes individuels, ce qui permet de capter l'essentiel des différences entre les individus. Cette méthode est également reprise par Givord et Wilner (2009) sur une période plus récente. Dans cette spécification, les effets individuels sont définis indépendamment les uns des autres, ce qui rend le nombre de paramètres à estimer très élevé. Une solution moins coûteuse revient à spécifier la forme de la distribution des effets individuels : on parle alors d'une modélisation de l'hétérogénéité inobservée par des effets aléatoires. C'est par exemple ce que fait Havet (2006). Ce ne sont alors que les paramètres de cette distribution qui doivent être estimés, ce qui permet de diminuer considérablement la difficulté de l'estimation. En outre,

2. La relation de travail est engagée pour une durée indéterminée mais peut-être rompue par l'employeur et/ou l'employé sans raison particulière et sans préavis. 
dans la modélisation à effets fixes, l'identification ne peut se faire que sur les personnes qui connaissent une transition entre deux états sur la période d'observation, ce qui peut être un problème si la « dépendance d'état 》 est différente entre les individus. La modélisation à effets aléatoires permet de diminuer cette difficulté ; la validité des résultats obtenus dépend cependant de la spécification retenue pour modéliser l'hétérogénéité inobservée.

S'ils permettent de bien prendre en compte l'hétérogénéité individuelle, les modèles précédents sont assez pauvres pour décrire la dynamique des trajectoires et la dépendance temporelle. En général, la dépendance à l'histoire passée se limite à la prise en compte de l'état occupé à la période précédente (processus de Markov d'ordre un). Cette spécification peut cependant être considérée comme trop frustre. Les trajectoires peuvent être plus durablement influencées par le fait d'avoir commencé sa carrière par tel ou tel emploi. La durée de ces épisodes et leur succession peuvent également influer sur la situation future. Lollivier (2000) montre ainsi que la durée d'un épisode de chômage est d'autant plus courte qu'elle n'a pas été précédée de périodes sans emploi auparavant. Sur la question spécifique des contrats courts, Calavrezo (2008) utilise un modèle triprobit, dont les différentes modalités dépendent de la durée des contrats temporaires. Les résultats ainsi obtenus vont dans le sens de l'hypothèse d'emploi temporaire comme tremplin en tout début de carrière. En revanche, l'impact du passage par l'emploi temporaire plus tardivement n'est plus aussi clair, la probabilité d'accès à l'emploi stable cinq ans après la sortie des études diminuant à mesure que l'individu reste dans un emploi temporaire.

Plus complets, les modèles « multi-états multiépisodes », que nous utilisons ici, modélisent simultanément durées des épisodes et transitions entre états, et permettent théoriquement de mieux prendre en compte cette dynamique. Bonnal et al. (1997) proposent ainsi un modèle complet de ces estimations. Le prix à payer pour ces modèles est que leur complexité les rend très lourds à estimer, ce qui explique qu'ils ont été peu mobilisés jusqu'à la mise à disposition récente de capacités de calculs nettement plus puissantes. Sur les dernières années, plusieurs travaux ont utilisé ce type de modélisation : citons ainsi Zijl et al. (2004) pour la Suède, Garcia Pérez et Muñoz-Bullon (2007) pour l'Espagne, Doiron et Gørgens (2008) pour l'Australie et pour la Belgique : Goebel et Verhofstadt (2008) et Cockx et Picchio (2009).
Cockx et Picchio concluent, en accord avec Doiron et Gørgens, que les passages par le chômage et l'emploi affectent les trajectoires futures sur le marché du travail. Les dépendances de durées passées semblent par contre jouer pour les jeunes Belges en difficulté, mais pas pour les jeunes Australiens. Sur des données italiennes, Gagliarducci (2005) est le premier à mettre en évidence des phénomènes non linéaires dans le temps : la probabilité qu'un emploi temporaire soit transformé en CDI est très importante dans un premier temps, mais s'atténue rapidement (ce qu'on peut interpréter comme un usage de certains CDD comme des périodes d'essai). Les personnes qui occupent des contrats temporaires à répétition, surtout lorsqu'ils s'accompagnent d'interruptions d'emploi, ont une probabilité plus faible de trouver un emploi stable. Beffy et al. (2008) se concentrent sur ce possible enfermement dans les trajectoires précaires pour les publics plus âgés que ceux qui nous intéressent ici. Ils estiment pour cela un modèle qui repose sur un mélange de chaînes de Markov, et distinguent les individus confinés entre l'emploi instable et le non-emploi de ceux qui peuvent accéder à un emploi stable.

\section{Les transitions entre emploi stable, emploi temporaire, chômage et inactivité entre 1998 et 2003}

$\mathbf{L}$ 'échantillon utilisé contient 2852 individus (cf. encadré 1). L'âge moyen en 2003 est un peu supérieur à 29 ans (cf. tableau 1). Plus de la moitié de notre échantillon a un diplôme de niveau supérieur ou égal au baccalauréat. Rappelons que nous conservons les personnes ayant entre un et six ans d'ancienneté en 1998, les proportions aux différentes durées d'expérience professionnelle sont donc presque identiques. Plus de la moitié de l'échantillon a changé de situation professionnelle au moins une fois entre mai 1998 et mai 2003. La majorité de l'échantillon occupe en emploi stable dès mai 1998.

\section{La situation de mai 2003 selon la situation cinq ans auparavant}

La répartition entre emploi stable, emploi temporaire, chômage et inactivité en 2003 présente des différences notables avec celle de 1998 (cf. tableau 1). Alors que la proportion de chômeurs 
est semblable en 1998 et 2003, quoique légèrement plus faible en 2003, la part des individus en emploi stable augmente de près de 20 points au détriment de celles des inactifs ou employés en contrat temporaire qui diminuent de 5 et 12 points respectivement. Les transitions à cinq ans conditionnellement à la situation en 1998 (dernière partie du tableau 1) permettent de

Encadré 1

\section{SOURCE ET DONNÉES}

Nous utilisons pour cette étude l'enquête Formation et Qualification Professionnelle de 2003. Cette enquête conduite par l'Insee fournit des données détaillées sur la formation scolaire, post-scolaire et continue, ainsi que sur le parcours professionnel des personnes interrogées. En plus des informations sur la situation professionnelle au moment de l'enquête et cinq ans auparavant, l'enquête de 2003 comporte un calendrier professionnel dans lequel sont reportés tous les changements de situation professionnelle survenus entre mai 1998 et mai 2003. Ce calendrier mensuel, qui ne figurait pas dans les précédentes enquêtes $F Q P$, est un outil précieux pour étudier les transitions sur le marché du travail. II décrit notamment les emplois occupés, les types de contrats signés, les dates et circonstances de début et de fin des épisodes d'emploi. Pour ce qui est des revenus, seuls les salaires perçus en 2002 sont reportés. Compte tenu des informations disponibles dans l'enquête, nous pouvons reconstituer les transitions faites sur le marché du travail entre mai 1998 et mai 2003. Nous nous reposons sur le type de contrat pour définir la nature temporaire ou stable de l'emploi. Nous utilisons également la durée des contrats et les transitions entre les différents types d'emploi pour préciser la qualité d'insertion professionnelle.

La situation professionnelle en mai 1998 et mai 2003 est renseignée à un niveau détaillé. On connaît notamment la nature du contrat occupé à chacune de ces deux dates, le secteur d'activité, la taille de l'entreprise ou encore le temps de travail au cas où l'individu est en emploi. En revanche, pour les autres périodes du calendrier, s'il est possible de distinguer les $C D I$ des CDD et missions intérim, les emplois aidés ne peuvent être différenciés des autres emplois temporaires. Les emplois aidés sont en général très mal repérés dans les enquêtes ménages, sans doute parce que certains d'entre eux consistent essentiellement en des aides fiscales pour les employeurs, dont les salariés concernés ne sont pas toujours conscients. Par exemple, Givord (2006) montre pour une autre enquête ménage, l'enquête Emploi de mars 2002, qu'elle permettait de repérer moins de $20 \%$ des emplois aidés du secteur marchand comptabilisés sur la même période par des sources administratives. Ainsi, il aurait été illusoire de tenter de détailler ce type d'emploi dans un calendrier rétrospectif. De même, ne sont pas disponibles les secteurs d'activité des entreprises fréquentées entre mai 1998 et mai 2003, ou leur appartenance au secteur public ou privé. Les alternances d'emploi très courts et de chômage sont décrites sans plus de détail dans l'enquête, et les passages de chômage à inactivité et vice-versa ne sont pas repérables. On sait si à l'issue d'un emploi l'individu entre au chômage ou devient inactif, mais on ne sait pas s'il reste dans cet état jusqu'à ce qu'il retrouve un emploi. Ces manques de précision renvoient au problème plus général des effets de mémoire dans les enquêtes rétrospectives, qui peuvent peser sur la qualité des données obtenues. II est probable que les emplois les plus anciens ou que les petits emplois courts sont moins décrits. Si notre exploitation du calendrier nous laisse penser que les données collectées sont assez précises, il subsiste un risque que nos résultats minorent certaines transitions.

Un atout de l'enquête de 2003 est la taille conséquente de son échantillon (près de 40000 répondants). Ceci nous permet de nous limiter à une sous-population homogène, les personnes en début de vie active. Plus précisément, nous nous concentrons sur les personnes ayant de un à six ans d'ancienneté au début de la période d'observation (mai 1998) et qui ont donc terminé leurs études initiales entre 1993 et 1997 . Nous ne conservons pas les jeunes qui ont moins d'un an d'ancienneté sur le marché du travail, ces débuts de carrières pouvant être très particuliers (contraintes de service national, stages de fin d'études...). Une sélection plus drastique aurait consisté à se limiter aux seuls sortants du système éducatif. Cela réduisait cependant très nettement la taille de notre échantillon (qui ne serait plus que de 500 individus), au risque de peser sur la qualité de nos estimations. Par ailleurs, s'intéresser à un échantillon plus large permet de mesurer les effets sur plus long terme de l'insertion. Lopez (2004) montre ainsi la longueur des processus d'insertion à partir de l'enquête Génération 98 du Céreq.

Des restrictions supplémentaires sont faites sur notre échantillon de base. Nous retirons ainsi les personnes de plus de 40 ans $(0,8 \%$ des 3452 individus ayant un à six ans d'expérience professionnelle en 1998), les militaires (3,9\% de l'échantillon de base), les individus qui se déclarent en contrat d'apprentissage ou aides familiales en 1998 ou 2003 (respectivement 1,6 \% et $0,5 \%$ de l'échantillon de base). Nous excluons également les individus qui ont commencé à travailler avant la fin de leur formation initiale $(9,9 \%$ de l'échantillon initial), cette restriction étant nécessaire pour que notre mesure d'expérience professionnelle, définie classiquement comme étant le nombre d'années écoulées depuis la sortie des études, ait un sens. Nous excluons enfin les individus pour lesquels les situations en début ou fin du calendrier sont manquantes, qui n'ont pas rempli le calendrier sans raison apparente $(4,6 \%$ de notre échantillon initial ont connu au moins un changement de situation entre 1998 et 2003 mais ne renseignent pas le calendrier) ou pour lesquels le calendrier recèle des incohérences (près de $7 \%$ reportent des dates de transitions incohérentes entre elles).

Au total, l'échantillon utilisé contient 2852 individus. 
comprendre ces évolutions. Il apparaît que près des deux tiers des personnes au chômage en mai 1998 occupent un emploi cinq ans plus tard, $15 \%$ ayant un emploi court et $48 \%$ bénéficiant d'un emploi de type stable. Le taux brut d'accès à l'emploi au bout de cinq ans est nettement plus élevé parmi celles qui étaient en emploi temporaire en 1998 : trois sur cinq ont trouvé un emploi stable ; une sur cinq occupe encore un emploi temporaire en 2003 et une sur cinq est au chômage ou inactive. Enfin, les emplois en CDI se révèlent de fait les meilleurs gages d'insertion puisque $88,4 \%$ occupent toujours un emploi de ce type au bout de cinq ans. Les personnes en emploi, même temporaire, en début de carrière sont donc plus souvent en emploi stable cinq ans après que les chômeurs.

Les chômeurs et occupants d'emploi temporaires en début de carrière sont différents en termes de caractéristiques observées (cf. tableau 1) : les individus en emploi court en mai 1998 sont plus diplômés, plus souvent des hommes et sont depuis plus longtemps sur le marché du travail que les chômeurs de 1998. Il est aussi probable que les populations de chaque état diffèrent dans des dimensions inobservables. Ces différences de caractéristiques pourraient à elles seules expliquer les différences observées à l'horizon de cinq ans. Il importe donc de contrôler ces effets de compositions pour identifier l'impact du passé professionnel sur l'insertion.

\section{Le processus d'insertion sur cinq ans}

Au-delà de l'état finalement occupé en 2003, la continuité des épisodes d'emploi et la persistance des épisodes de chômage sont deux indicateurs importants de la qualité de l'insertion professionnelle des jeunes. Il existe une grande diversité des trajectoires sur cinq ans au sein de la population étudiée (cf. tableau 2). Les hommes ont des trajectoires plus stables et restent en

Tableau 1

Composition de l'échantillon en 2003

\begin{tabular}{|c|c|c|c|c|c|}
\hline & \multirow[b]{2}{*}{ Échantillon total } & \multicolumn{4}{|c|}{ Selon la situation de mai 1998 : } \\
\hline & & Chômage & Inactivité & $\begin{array}{c}\text { Emploi } \\
\text { temporaire }\end{array}$ & Emploi stable \\
\hline $\begin{array}{l}\text { Nombre d'observations } \\
\text { En \% }\end{array}$ & $\begin{array}{r}2852 \\
100\end{array}$ & $\begin{array}{l}320 \\
11,2\end{array}$ & $\begin{array}{l}400 \\
14\end{array}$ & $\begin{array}{l}647 \\
22,7\end{array}$ & $\begin{array}{c}1485 \\
52,1\end{array}$ \\
\hline Femmes (en \%) & 54,6 & 59,4 & 73,5 & 53,3 & 49 \\
\hline Français (en \%) & 95,7 & 96,6 & 86,8 & 97,8 & 96,9 \\
\hline Âge moyen & 29,3 & 28,2 & 28,1 & 28,7 & 30,1 \\
\hline \multicolumn{6}{|l|}{ Diplôme (en \%) } \\
\hline $\begin{array}{l}\text { Aucun ou CEP } \\
\text { BEPC seul } \\
\text { CAP, BEP ou équivalent } \\
\text { Bac, brevet prof. ou équivalent } \\
\text { Bac }+2 \text { ans } \\
\text { Diplôme supérieur }\end{array}$ & $\begin{array}{c}11,9 \\
5 \\
17,3 \\
23,7 \\
19,5 \\
22,6\end{array}$ & $\begin{array}{r}23,4 \\
9,7 \\
18,1 \\
22,8 \\
11,6 \\
14,4\end{array}$ & $\begin{array}{r}24,8 \\
5,5 \\
17,8 \\
21 \\
10,5 \\
20,5\end{array}$ & $\begin{array}{c}11,1 \\
6 \\
22,1 \\
26,7 \\
15,8 \\
18,2\end{array}$ & $\begin{array}{r}6,3 \\
3,4 \\
14,9 \\
23,2 \\
25,3 \\
26,8\end{array}$ \\
\hline \multicolumn{6}{|c|}{ Nombre d'années d'expérience en 1998 (en \%) } \\
\hline $\begin{array}{l}1 \\
2 \\
3 \\
4 \\
5\end{array}$ & $\begin{array}{l}16,7 \\
19,2 \\
21,3 \\
22,5 \\
20,3\end{array}$ & $\begin{array}{l}24,1 \\
18,4 \\
19,7 \\
22,2 \\
15,6\end{array}$ & $\begin{array}{l}24,8 \\
20 \\
19 \\
18,5 \\
17,8\end{array}$ & $\begin{array}{l}21,3 \\
23,5 \\
21,9 \\
19,2 \\
14,1\end{array}$ & $\begin{array}{l}10,9 \\
17,4 \\
22 \\
25,1 \\
24,7\end{array}$ \\
\hline \multicolumn{6}{|c|}{ Nombre d'épisodes entre mai 1998 et mai 2003 (en \%) } \\
\hline $\begin{array}{l}1 \\
2 \\
3 \text { et plus }\end{array}$ & $\begin{array}{l}44,7 \\
14,1 \\
41,2\end{array}$ & $\begin{array}{l}10,6 \\
28,1 \\
61,3\end{array}$ & $\begin{array}{l}25,5 \\
25,5 \\
49\end{array}$ & $\begin{array}{l}10,8 \\
22,6 \\
66,6\end{array}$ & $\begin{array}{r}72,1 \\
4,2 \\
23,7\end{array}$ \\
\hline \multicolumn{6}{|l|}{ Situation en mai 2003 (en \%) } \\
\hline $\begin{array}{l}\text { Chômage } \\
\text { Inactivité } \\
\text { Emploi précaire } \\
\text { Emploi stable }\end{array}$ & $\begin{array}{r}8,8 \\
8,8 \\
10,8 \\
71,6\end{array}$ & $\begin{array}{r}29,7 \\
7,2 \\
15,3 \\
47,8\end{array}$ & $\begin{array}{l}9,5 \\
38,5 \\
10 \\
42\end{array}$ & $\begin{array}{r}9,4 \\
5,1 \\
22,4 \\
63,1\end{array}$ & $\begin{array}{c}3,9 \\
2,7 \\
5 \\
88,4\end{array}$ \\
\hline
\end{tabular}

Lecture : deux tiers des personnes au chômage en mai 1998 occupent un emploi cinq ans plus tard, 15 \% occupent un emploi court et $48 \%$ un emploi de type stable.

Champ : individus ayant moins de 10 ans d'expérience professionnelle en mai 2003.

Source: FQP 2003, Insee. 
moyenne plus longtemps en emploi stable que les femmes : près de la moitié des hommes ne changent pas de situation professionnelle sur les cinq ans, alors que $44,5 \%$ des femmes ont connu au moins trois épisodes sur le marché du travail. Le nombre d'épisodes sur cinq ans décroît avec l'ancienneté sur le marché du travail en 1998. Plus la personne était expérimentée en 1998, et plus la part du temps passé en moyenne en emploi stable augmente tandis que celle passée en emploi temporaire diminue. Ce fait stylisé est cohérent avec un schéma d'insertion progressif, décrit par exemple par Lopez (2004). Il en est de même pour le niveau de diplôme, même si l'effet n'est pas linéaire. Plus de la moitié des individus avec un diplôme de niveau supérieur au baccalauréat sont restés dans le même état entre mai 1998 et mai 2003, tandis que 62,2 \% des détenteurs d'un BEPC ont subi au moins trois épisodes sur le marché du travail. Enfin, 72,1\% des individus en emploi stable en 1998 le sont restés sur les cinq ans. Les personnes en emploi court en 1998 semblent légèrement plus mobiles que celles qui étaient au chômage à la même date : $66,6 \%$ des occupants d'emploi temporaire ont changé au moins trois fois de situation professionnelle, contre $61,3 \%$ des personnes au chômage en 1998. Si les chômeurs et salariés qui étaient en emploi temporaire en 1998 ont passé en moyenne chacun un peu moins d'un quart des 60 mois d'observation en emploi stable, les premiers sont restés en moyenne plus longtemps hors de l'emploi que les seconds sur l'ensemble de la période.

\section{Les « épisodes » sont de durées différentes selon les caractéristiques des personnes}

Les hommes qui occupent un emploi temporaire connaissent plus rapidement que les femmes une transition vers un emploi stable (cf. graphique I). Cette différence entre hommes est femmes est encore plus forte lorsque l'on considère les sorties vers l'emploi stable à partir du nonemploi (cf. graphique II). Ce résultat semble en grande partie dû aux moindres sorties de l'inactivité vers l'emploi chez les femmes (3).

Pour mettre en évidence la corrélation entre la trajectoire passée et l'accès à l'emploi stable, nous présentons également les durées des épisodes d'emploi temporaire et de chômage, avant de connaître une transition vers l'emploi

3. Les différences entre hommes et femmes sont plus faibles et moins significatives lorsque l'on se restreint aux sorties de chômage (dans ce cas, seul le test de ratio des vraisemblances conduit à rejeter l'hypothèse nulle d'égalité des strates).

Tableau 2

Résumé des trajectoires individuelles sur cinq ans

\begin{tabular}{|c|c|c|c|c|c|c|}
\hline & \multicolumn{3}{|c|}{ Nombres d'épisodes } & \multicolumn{3}{|c|}{ Nombre de mois en ... : } \\
\hline & 1 & 2 & 3 ou plus & $\begin{array}{c}\text { Emploi } \\
\text { temporaire }\end{array}$ & Emploi stable & Non-emploi \\
\hline Hommes & 48,5 & 14,3 & 37,3 & 10,8 & 42,2 & 7,1 \\
\hline Femmes & 41,6 & 13,9 & 44,5 & 10,8 & 34,4 & 14,9 \\
\hline \multicolumn{7}{|c|}{ Nombre d'années d'expérience en 1998} \\
\hline 1 & 27,9 & 17,2 & 54,8 & 15 & 32 & 13,1 \\
\hline 2 & 39,7 & 17,1 & 43,2 & 12,2 & 37 & 10,9 \\
\hline 3 & 47 & 12,5 & 40,5 & 10,2 & 38,5 & 11,4 \\
\hline 4 & 49,8 & 13,4 & 36,8 & 9,5 & 39,7 & 10,8 \\
\hline 5 & 55,4 & 10,9 & 33,7 & 7,9 & 41,3 & 10,9 \\
\hline \multicolumn{7}{|l|}{ Diplôme } \\
\hline Aucun ou CEP & 37,2 & 13 & 49,9 & 12,2 & 21,6 & 26,3 \\
\hline BEPC seul & 24,5 & 13,3 & 62,2 & 16 & 26,3 & 18 \\
\hline CAP, BEP ou éq. & 34 & 13,4 & 52,6 & 13,4 & 34 & 12,7 \\
\hline Bac, brevet prof. ou éq. & 41,3 & 14,8 & 43,9 & 11,9 & 37,9 & 10,2 \\
\hline $\mathrm{Bac}+2$ ans & 58,3 & 11,7 & 30 & 7,8 & 47,2 & 5 \\
\hline Diplôme supérieur & 53,3 & 16,6 & 30,1 & 8,2 & 44,3 & 7,6 \\
\hline \multicolumn{7}{|l|}{ Situation en mai 1998} \\
\hline Chômage & 10,6 & 28,1 & 61,3 & 13,1 & 21,8 & 25,3 \\
\hline Inactivité & 25,5 & 25,5 & 49 & 8,6 & 15,1 & 36,4 \\
\hline Emploi stable & 72,1 & 4,2 & 23,7 & 2,8 & 54,3 & 2,9 \\
\hline Emploi temporaire & 10,8 & 22,6 & 66,6 & 29,2 & 22,7 & 8,3 \\
\hline
\end{tabular}

Lecture : $62 \%$ des détenteurs d'un seul BEPC ont connu 3 épisodes ou plus sur le marché du travail. Champ : individus ayant moins de 10 ans d'expérience professionnelle en mai 2003.

Source : FQP 2003, Insee. 
stable, selon l'état occupé à l'épisode précédent. Il s'agit de capter d'éventuels effets sur le long terme de la trajectoire passée. Les transitions entre emploi temporaire et emploi stable sont plus rapides lorsque la personne occupait précédemment un emploi stable plutôt que temporaire, ou un emploi temporaire plutôt que

\section{Graphique I}

Durée en emploi temporaire avant une transition vers un emploi stable par sexe (estimateur de Kaplan-Meier)

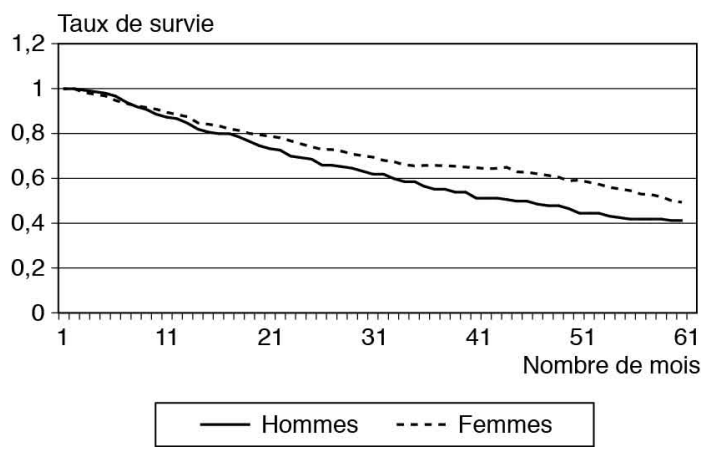

Lecture : estimation non paramétrique de la fonction de survie dans l'emploi temporaire jusqu'à une transition vers un emploi stable. $80 \%$ des hommes ayant survécu en emploi temporaire pendant 15 mois obtiennent un emploi stable au $16^{\mathrm{e}}$ mois. Test du log-rank d'égalité des fonctions de survie dans l'emploi temporaire : par sexe chi2(1) =6,6; soit rejet de l'homogénéité des strates pour un test à $5 \%$.

Champ : épisodes d'emploi temporaires réalisés par les individus ayant moins de 10 ans d'expérience professionnelle en mai 2003. Nombre d'épisodes utilisés : 1290 épisodes pour les femmes et 1442 épisodes pour les hommes. Source : FQP 2003, Insee.

\section{Graphique III}

Durée en emploi temporaire avant une transition vers un emploi stable selon l'état occupé précédemment (estimateur de Kaplan-Meier)

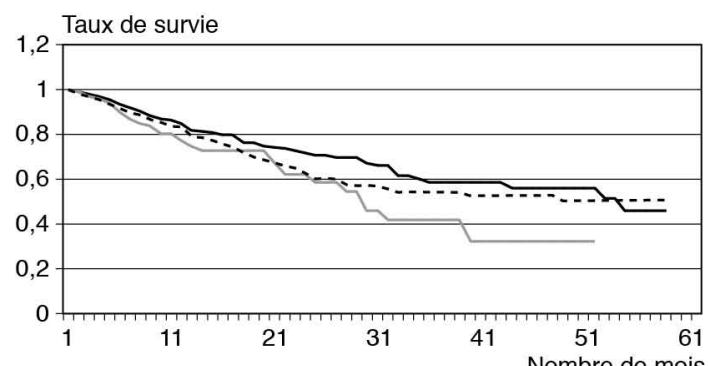

$\begin{array}{lll}\text { - État précédent stable } & \text {--- État précédent précaire } \\ \text { — État précédent non-emploi } & \end{array}$

Lecture : Estimation non paramétrique de la fonction de survie. $73 \%$ des individus en emploi temporaire depuis 15 mois et qui étaient en emploi stable lors de l'épisode précédent obtiennent un emploi stable au $16^{e}$ mois. Test du log-rank d'égalité des fonctions de survie dans l'emploi temporaire : par situation précédente chi2(1) = 7,7 ; soit rejet de l'homogénéité des strates pour un test à $5 \%$.

Champ : épisodes d'emploi temporaires commencés au cours du calendrier et réalisés par les individus ayant moins de 10 ans d'expérience professionnelle en mai 2003. Nombre d'épisodes utilisés : 1094 épisodes pour les femmes et 982 épisodes pour les hommes.

Source : FQP 2003, Insee. lorsqu'elle était hors de l'emploi (cf. graphique III). De même, les personnes sans emploi accèdent plus vite à un emploi stable lorsqu'elles occupaient précédemment un emploi stable plutôt qu'un emploi temporaire (cf. graphique IV). Ces différences ne sont que faiblement significatives. En tout état de cause, il n'est pas

\section{Graphique II \\ Durée hors de l'emploi avant une transition vers un emploi stable par sexe (estimateur de Kaplan-Meier)}

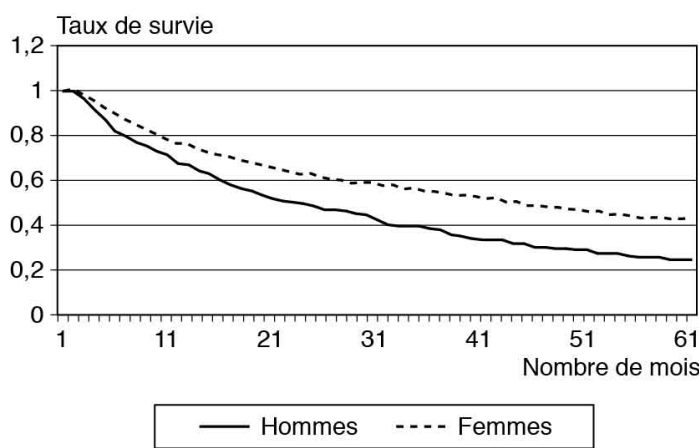

Lecture : estimation non paramétrique de la fonction de survie hors de l'emploi jusqu'à une transition vers un emplo stable. $56 \%$ des hommes restés hors de l'emploi pendant 15 mois obtiennent un emploi stable au $16^{e}$ mois. Test du logrank d'égalité des fonctions de survie au chômage : par sexe chi2(1) = 30,8; soit rejet de l'homogénéité des strates pour un test à $1 \%$.

Champ : épisodes de non-emploi réalisés par les individus ayant moins de 10 ans d'expérience professionnelle en mai 2003. Nombre d'épisodes utilisés : 1509 épisodes pour les femmes et 972 épisodes pour les hommes.

Source : FQP 2003, Insee.

Graphique IV

Durée hors de l'emploi avant une transition vers un emploi stable selon l'état occupé précédemment (estimateur de Kaplan-Meier)

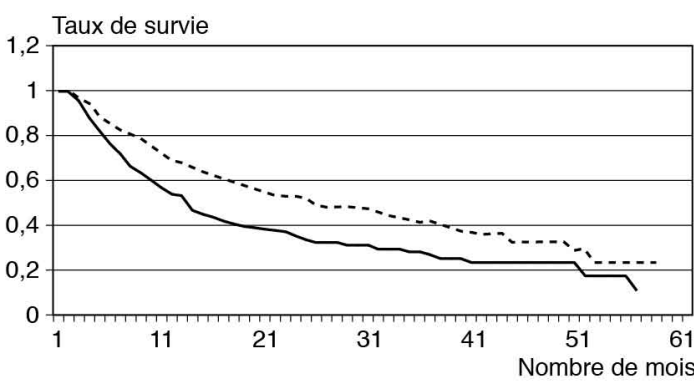

— État précédent stable - - - - État précédent précaire

Lecture : estimation non paramétrique de la fonction de survie hors de l'emploi jusqu'à une transition vers un emploi stable. $43 \%$ des individus non occupés depuis 15 mois et qui étaient en emploi stable lors de l'épisode précédent obtiennent un emploi stable au $16^{e}$ mois. Test du log-rank d'égalité des fonctions de survie hors de l'emploi : par situation précédente chi2(2) = 37,8 soit rejet de l'homogénéité des strates pour un test à $1 \%$.

Champ : épisodes de non-emplois réalisés commencés au cours du calendrier et réalisés par les individus ayant moins de 10 ans d'expérience professionnelle en mai 2003. Nombre d'épisodes utilisés : 1025 épisodes pour les femmes et 700 épisodes pour les hommes.

Source : FQP 2003, Insee. 
possible d'en conclure à ce stade que ces effets ne soient pas liés à des composantes inobservées : les personnes qui occupaient des emplois moins insérés dans le passé peuvent avoir des caractéristiques inobservables qui rendent également moins probable l'accès à un emploi stable dans le futur. Il reste donc à déterminer ce qui relève vraiment de la dépendance d'état (i.e. les effets durables de la trajectoire passée) de ces effets de composition.

\section{Une modélisation simultanée des durées et des transitions}

P our estimer la dépendance dans un état, nous utilisons un modèle de hasards à plusieurs périodes avec plusieurs états et risques concurrents. Un individu en emploi temporaire peut en sortir pour être au chômage ou pour un emploi de type temporaire ou stable. L'obtention d'un emploi peut prendre plus ou moins de temps et il peut être plus ou moins aisé de trouver un emploi stable qu'un emploi court selon les caractéristiques individuelles. En particulier, avoir déjà connu plusieurs épisodes de chômage, être resté longtemps au chômage, le niveau de diplôme et le temps passé dans l'emploi temporaire peuvent affecter simultanément la probabilité d'accès à l'emploi stable à partir d'un emploi temporaire. Les modèles à risques concurrents prennent en compte cette multiplicité de possibilités et le fait que de nombreux éléments viennent déterminer la probabilité de sortie vers un état plutôt qu'un autre (cf. encadré 2).

En prenant un modèle à hasards proportionnels mixtes (Lancaster, 1990 ; van den Berg, 2001), il est possible de dissocier l'effet des caractéristiques individuelles observées et inobservées de l'individu de la dépendance temporelle (la probabilité de sortie d'un état varie en fonction du temps déjà passé dans cet état) et de la dépendance d'état (la probabilité d'accéder à l'emploi stable est plus ou moins grande selon que l'on est en emploi temporaire ou au chômage). Il est également possible d'isoler l'effet des dépendances passées sur les probabilités instantanées d'entrer dans un nouvel état (Borjas et Heckman, 1980 ; Lollivier, 2000 ; Horny et Picchio, 2009). C'est par le biais des covariables que nous tenons compte du possible impact du passé sur les transitions professionnelles.

Encadré 2

\section{MODÈLE À RISQUES CONCURRENTS AVEC MULTI-ÉPISODES, HÉTÉROGÉNÉITÉ INOBSERVÉE, DÉPENDANCES PASSÉES ET PROBLĖME DE CONDITIONS INITIALES}

\section{Modèle à risques concurrents}

Pour chaque individu, on observe sur 60 mois le nombre de transitions opérées sur le marché du travail, les états occupés successivement et le temps passé dans chaque épisode. Pour modéliser cet historique, on définit des processus latents $U_{j k} \in R^{+}$, où $j=1,2,3$ désigne l'état d'origine, et $k$ un état de destination possible à partir de l'état $j: k \in E(j)$ où $E(j)$ est l'ensemble des états accessibles à partir de $j$. Les processus ainsi définis correspondent à la durée nécessaire pour sortir de l'état $j$ et entrer dans l'état $k$. Si l'individu sort de l'état $j$, la transition effectivement observée correspond à celle pour laquelle la durée latente est la plus courte. Ainsi la durée de l'épisode est donnée par la plus petite des valeurs prises par la variable $U_{j k^{\prime}}$ Si l'individu est toujours en $j$ à la fin de la période d'observation, le processus est dit censuré. L'information dont on dispose alors est que le temps nécessaire pour atteindre chacun des états $k \in E(j)$ est supérieur au temps observé.

On considère que quelle que soit la transition $j k$ considérée, les différences observées entre les individus dans la fonction de hasard de $u_{j k}$, la réalisation de $U_{j k}$, peuvent être caractérisées par les variables observées $X$ et inobservées $v_{j k^{\prime}}$. Notamment, on suppose que quels que soient $k, k^{\prime} \in E(j)$, les durées $u_{j k}$ et $u_{j k^{\prime}}$ sont indépendantes conditionnellement à X, $v_{j k}, v_{j k^{\prime}}$.

Notre calendrier nous permet d'observer pour une même personne $i$ ses $L_{i}$ transitions successives sur le marché du travail. On s'intéresse ici à la distribution jointe des durées conditionnellement aux observables $f\left(u_{2}, \ldots, u_{L_{i}} \mid X\right)$ (par souci de clarté, on omet les indices $j$ et $k$ ). Sous l'hypothèse d'indépendance conditionnelle sur les observables et inobservables, il est possible de décrire la densité jointe conditionnelle comme le produit des densités marginales conditionnelles de chacune des durées $u_{l}$ (avec $I=2, \ldots, L_{i}$ ) intégré sur la distribution des termes inobservables.

Dans un cadre de risques concurrents, les données apportent de l'information sur $\min \left(u_{j k}, u_{j k^{\prime}}\right)$ et sur la durée $U_{j k}$ se terminant en premier. Comme ces informations ne sont pas suffisantes pour permettre l'identification du modèle (Lancaster, 1990), nous devons imposer une structure sur les hasards. L'intensité de transition vers l'état $k$ après avoir passé une durée $u$, dans l'état $j$ est ainsi supposée de type hasard proportionnel mixte. Selon cette spécifica- 


\section{Encadré 2 (suite)}

tion, les variables explicatives et l'hétérogénéité inobservable font varier le hasard de base proportionnellement. La fonction de hasard conditionnel du lème épisode s'écrit donc

$h_{j k}\left(u_{l} \mid X_{j k}\left(\tau_{l}\right), v_{j k}\right)=h_{j k}^{o}\left(u_{l}\right) \psi\left[X_{j k}\left(\tau_{l}\right)^{\prime} \beta_{j k}+v_{j k}\right]$

où $X_{j k}(\tau)$ représentent les caractéristiques observables de l'individu, dont l'état occupé précédemment. Leur valeur est fixée à la date $\tau$, d'entrée dans l'épisode. $v_{\mathrm{jk}}$ capte l'effet de l'hétérogénéité individuelle inobservée sur la transition de l'état $j$ vers l'état $k$. On autorise cette hétérogénéité à dépendre de l'état d'origine $j$ et de destination $k$. Nous supposons cependant que ces caractéristiques individuelles inobservables sont constantes sur l'ensemble de la trajectoire professionnelle. On suppose ainsi par exemple que le passé professionnel n'affecte pas la motivation ou l'attachement au marché du travail. Enfin, $h_{j k}^{o}($.$) est la fonction de hasard de base : elle décrit comment la proba-$ bilité de sortie d'un état dépend du temps déjà passé dans cet état et mesure donc la dépendance de durée. On suppose que cette fonction peut dépendre de l'état d'origine $j$ et de l'état d'arrivée $k$, mais qu'elle ne dépend pas du rang / de l'épisode actuel dans l'histoire des transitions. Cette hypothèse signifie que le passé professionnel ne joue pas sur la dépendance de durée.

L'identification de ce type de modèle est dite non paramétrique dans la mesure où elle ne repose pas sur des formes fonctionnelles spécifiques de $\psi$, du hasard de base et de la distribution des termes inobservés. Le fait de disposer de multiples épisodes par individus nous permet d'avoir une identification sous des hypothèses plus faibles que lorsque l'on n'observe qu'une réalisation par individu (voir Abbring et Van den Berg, 2003 pour les conditions précises d'identification du modèle).

Pour la partie spécifique $\psi$ nous prenons classiquement une fonction exponentielle. Pour le hasard de base, plusieurs spécifications peuvent être retenues : aucune n'est a priori évidente, et choisir une spécification paramétrique $a d$ hoc pour cette fonction peut conduire à des résultats erronés, si la spécification retenue ne correspond pas à la forme réelle de la dépendance de durée. Pour cette raison, nous préférerons utiliser une spécification flexible, en approximant la fonction par des hasards de base constants par morceaux (avec des intervalles de 3, 6 puis 12 mois) :

$h_{j k}^{o}\left(u_{l}\right)=\exp \left(\sum_{s=1}^{M} \alpha_{j k}^{s} 1\left(u_{l} \in[s-1 ; s]\right)\right)$

\section{Vraisemblance conditionnelle de l'échantillon}

Nous décrivons ici les contributions individuelles à la vraisemblance, conditionnellement à la condition initiale $\mathrm{y}_{1}=1,2,3$ et à l'hétérogénéité inobservée. Du fait de notre traitement de la condition initiale, seuls les épisodes commencés postérieurement à mai 1998, soient les épisodes 2 à $L_{i}$, contribuent à la vraisemblance.

Si le lème épisode $\left(I=2, \ldots, L_{i}\right)$ n'est pas censuré à droite (c'est-à-dire s'il est terminé à la date de l'enquête en mai 2003) et marque une transition de $j$ vers $k$, sa contribution à la vraisemblance est la fonction de densité conditionnelle de la durée passée dans l'état $j$, étant donné que l'individu était dans l'état $y_{1}$ en mai 1998, que l'épisode dure $u$, mois et se termine avec une transition vers l'état $k$ :

$f\left(u_{l} \mid y_{1}, X_{j k}\left(\tau_{l}\right), v_{j k}\right)=h_{j k}\left(u_{l} \mid y_{1}, X_{j k}\left(\tau_{l}\right), v_{j k}\right) S_{j}\left(u_{l} \mid y_{1}, X_{j k}\left(\tau_{l}\right), v_{j k}\right)$

où $S_{j}(. \mid$.$) est la fonction de survie conditionnelle de la durée u$, dans l'état $j$, définie par :

$S_{j}\left(u_{l} \mid y_{1}, X_{j k}\left(\tau_{l}\right), v_{j k}\right)=\exp \left(-\sum_{k^{\prime} \in E(j)} \int_{s=0}^{u_{l}} h_{j k^{\prime}}\left(s \mid y_{1}, X_{j k^{\prime}}\left(\tau_{l}\right), v_{j k^{\prime}}\right) d s\right)$

Sa contribution à la vraisemblance est donc la probabilité $h_{j k}\left(u_{1} \mid y_{1}, X_{j k}(\tau), v_{j k}\right)$ que l'individu reste au moins $u_{1}$ mois dans l'état $j$ avant d'entrer dans l'état $k$, multipliée par la probabilité $S_{j}\left(u_{l} \mid y_{1}, X_{j k}(\tau), v_{j k}\right)$ qu'il n'ait pas connu de transition vers un autre état auparavant (c'est-à-dire que les durées pour sortir de $j$ vers un autre état $k$ soient supérieures à $\left.u_{1}\right)$.

Pour les épisodes censurés à la date de l'enquête en mai 2003 , la contribution à la fonction de vraisemblance est :

$f\left(u_{1} \mid y_{1}, X_{j k}(\tau), v_{j k}\right)=S_{j}\left(T-\tau_{l} \mid y_{1}, X_{j k}\left(\tau_{1}\right), v_{j k}\right)$

où $T$ la date de fin de l'observation (mai 2003).

C'est donc simplement la probabilité que la durée nécessaire pour sortir de l'état $j$ est supérieure à la durée observée $T-\tau_{l}$. 
La mesure de la dépendance d'état, courante ou passée, peut être fallacieuse. Par exemple, un jeune entrant sur le marché du travail pourvu d'une certaine motivation peut accepter un emploi court plutôt que de rester au chômage. Cette motivation peut par ailleurs lui permettre d'obtenir une conversion du contrat court en emploi permanent et de le garder durablement. Ignorer la sélection sur caractéristiques inobservables conduirait à surestimer l'impact du passé professionnel, puisque l'on attribuerait entièrement au passage par l'emploi temporaire une stabilité en emploi plus grande, ce qui serait erroné. Les données utilisées fournissent pour une même personne les différents épisodes de sa trajectoire professionnelle. Ceci permet théoriquement de modéliser une partie de l'hétérogénéité inobservée, et donc d'estimer l'effet réel de ces dépendances d'états.

Les données étant mensuelles, nous estimons des probabilités mensuelles de transitions. Bien que ces données soient discrètes, nous traitons les durées comme les réalisations d'un processus en temps continu à l'instar de van den Berg et van der Klaauw (2001). Nous nous intéressons ici aux transitions entre emploi stable, emploi temporaire et «non-emploi » (cf. tableau 3). Les transitions entre chômage et inactivité n'étant pas observables, chômage et inactivité sont agrégés dans « non-emploi ». Du fait de cette agrégation, et suivant les conclusions de Havet (2006) qui montre une diversité des parcours selon le genre, le modèle est estimé séparément pour les hommes et les femmes. Cette étude étant consacrée au processus d'insertion professionnelle, les transitions directes entre emplois stables ne sont pas prises en compte. Nous opérons ainsi une modélisation différente des transitions selon le type d'emploi considéré et ne modélisons les successions d'emploi de même type que s'ils sont temporaires. En ce sens, nous estimons la persistance en emplois de type stable, mais conservons la dimension contrat pour les emplois temporaires. Au final, les transitions modélisées sont de types emploi temporaire vers emploi temporaire, emploi stable ou non-emploi ; emploi stable vers emploi temporaire ou non-emploi ; et non-emploi vers emploi stable ou temporaire.

Encadré 2 (fin)

La contribution conditionnelle d'un individu à la vraisemblance correspond au produit de la vraisemblance conditionnelle de chacun de ses épisodes, sauf le premier. La vraisemblance conditionnelle de l'échantillon est le produit sur tous les individus et s'écrit donc :

$L\left(u_{2}, \ldots, u_{L_{i}} \mid y_{1}, X_{i}, v ; \Theta\right)=\prod_{i=1}^{n} I\left(u_{2}, \ldots, u_{L_{i}} \mid y_{1 i}, X_{i}, v ; \Theta\right)$

où la contribution individuelle $I\left(u_{2}, \ldots, u_{L_{i}} \mid y_{1 i}, X_{i}, v ; \Theta\right)$ est :

$I\left(u_{2}, \ldots, u_{L_{i}} \mid y_{1 i}, X_{i}, v ; \Theta\right)=\prod_{l=2}^{L_{i}} \prod_{j=1}^{3} \prod_{k \in E(j)} h\left(u_{l} \mid y_{1 i}, X_{j k i}, v_{j k}\right)^{\delta_{j k i}^{\prime}} S_{j}\left(u_{l} \mid y_{1 i}, X_{j k i}, v_{j k}\right)^{\delta_{j i}^{-l}}$

où $\delta_{j k i}^{\prime}=\left\{\begin{array}{l}1 \text { si l'épisodel est unetransition de } j \text { vers } k \\ 0 \text { sinon }\end{array}\right.$

et $\delta_{j i}^{l}=\sum_{k \in E(j)} \delta_{j k i}^{l}$.

Hétérogénéité inobservée

Pour obtenir la vraisemblance non conditionnelle aux $\left(v_{\mathrm{jk}}\right)_{\mathrm{j}}$ qui ne sont pas - par définition - observés, il est nécessaire d'intégrer par rapport à la distribution des termes inobservés. II est souvent d'usage dans la littérature de retenir une distribution discrète à la Heckman-Singer (1984). Cette formulation est a priori plus souple qu'une spécification paramétrique ad hoc de la distribution de l'hétérogénéité inobservée. Formellement, on suppose que $v=\left(v_{12}, \ldots, v_{32}\right)$ a une distribution discrète définie par les probabilités suivantes, estimées conjointement aux autres paramètres du modèle :

$P_{m}=\operatorname{Pr}\left(v=v^{m}=\left(v_{12}^{m}, \ldots, v_{32}^{m}\right)\right)$ où $0 \leq P_{m} \leq 1, \forall m=1, \ldots$, Met $\sum_{k=1}^{M} P_{m}=1$

Au final, la fonction de vraisemblance non conditionnelle est :

$L\left(u_{2}, \ldots u_{L} \mid y_{1}, X ; \Theta\right)=\prod_{i=1}^{n} \sum_{m=1}^{M} P_{m} /\left(u_{2}, \ldots, u_{L_{i}} \mid y_{1 i}, X_{i}, v^{m} ; \Theta\right)$. 
Pour mesurer l'impact causal du passage par l'emploi temporaire ou le chômage sur la trajectoire future, il faut tenir compte des effets de compositions observables et inobservables.

Pour tenir compte de l'hétérogénéité observable, les caractéristiques individuelles étudiées précédemment sont incluses dans le modèle. Certaines sont stables dans le temps, comme la nationalité et le niveau de diplôme ; d'autres sont variables, comme l'âge et la situation familiale. Dans ce cas, la valeur de la caractéristique prise en compte est celle correspondant au début de l'épisode considéré. Pour mesurer les dépendances d'état passées, l'état occupé durant l'épisode précédent est inclus dans l'ensemble des variables explicatives. La dépendance de durée actuelle est captée par les variations du hasard de base (cf. encadré 2). Enfin, pour tenir compte de la sélection sur variables inobservables, nous prenons une distribution multivariée pour les termes d'hétérogénéité inobservée et autorisons les termes spécifiques à chaque type de transition à être corrélés.

La trajectoire professionnelle des individus n'est observable que sur une période de cinq ans avant la date d'enquête, soit à partir de mai 1998. Tous les jeunes actifs de notre échantillon n'en sont pas au même point dans leur trajectoire professionnelle à leur entrée dans le calendrier : ils occupent des positions professionnelles différentes, et ce depuis plus ou moins longtemps. Ne pas tenir compte des conditions initiales risque de biaiser les estimations : l'état occupé au début du calendrier est le résultat d'une trajectoire antérieure que l'on n'observe pas et dont les déterminants sont corrélés avec ceux de la trajectoire observée. Pour en tenir compte, la méthode proposée par Wooldridge (2005) consiste à modéliser la distribution de l'hétérogénéité inobservée conditionnellement à la situation initiale, i.e. à ajouter les situations occupées en mai 1998, à la liste des variables explicatives ; on modélise ensuite la densité jointe des épisodes suivants (4). Ce traitement des conditions initiales implique que le modèle ne peut être estimé que sur les individus qui changent au moins une fois de situation sur le marché du travail au cours des cinq ans $(56,3 \%$ de notre échantillon). Dans leur très grande majorité (84\%), les personnes qui ne connaissent pas de transition entre 1998 et 2003 occupaient déjà un emploi stable en 1998.

\section{Des trajectoires moins favorables pour les jeunes femmes}

$\mathbf{L}$ e modèle économétrique multi-états multiépisodes nous permet de décrire l'impact des caractéristiques observables des personnes et de la dépendance de durée tout en tenant compte de l'hétérogénéité inobservée. Les résultats descriptifs suggèrent que les trajectoires professionnelles des hommes et des femmes peuvent être différentes. Aussi, nous avons procédé à des estimations séparées par genre.

La plupart des caractéristiques disponibles susceptibles d'affecter les intensités de transition d'un état à un autre ont été retenues pour l'analyse : outre l'âge et le diplôme, la nationalité (française ou pas), ainsi que la présence de jeunes enfants (moins de trois ans) dans le ménage au début de l'épisode (5). C'est sur cette

4. Il est à noter qu'en reconstruisant tout le processus de transitions de mai 1998 à la date l'échantillonnage en mai 2003, nous résolvons le problème de biais d'échantillonnage en stock qui pèse sur les épisodes en cours en mai 2003.

5. Le statut marital n'est pas inclus du fait de la difficulté rencontrée pour reconstituer son évolution au cours de la période.

Tableau 3

Matrice des transitions estimées

\begin{tabular}{|l|c|c|c|c|c|}
\hline $\begin{array}{l}\text { Destination } \rightarrow \\
\text { Origine }\end{array}$ & $\begin{array}{c}\text { Emploi } \\
\text { temporaire }\end{array}$ & Emploi stable & Non-emploi & Censuré & Total \\
\hline Emploi temporaire & 25,7 & 14,3 & 46,4 & 13,6 & 100 \\
& $(533)$ & $(297)$ & $(963)$ & $283)$ & $(2076)$ \\
Emploi stable & 5,2 & - & $(259)$ & $(927)$ & 1 \\
& $(65)$ & - & - & 20,8 & $(1251)$ \\
Non-emploi & 48,4 & 30,8 & - & $(366)$ & 100 \\
& $(852$ & $(543)$ & & $(1761)$ \\
\hline
\end{tabular}

Lecture : 14,3\% des 2076 épisodes commençant par un emploi temporaire finissent par un emploi stable; 13,6 \% sont toujours en cours au moment de l'enquête (ils sont dit censurés).

Champ : 5088 épisodes commencés au cours du calendrier.

Source : FQP 2003, Insee. 
dimension que les contrastes sont les plus marqués entre hommes et femmes (cf. tableaux 4 et 5). La présence de jeunes enfants fragilise la situation professionnelle des jeunes femmes. Les transitions entre emploi temporaire et emploi stable sont ainsi considérablement diminuées, alors que les transitions vers le nonemploi (chômage ou inactivité) sont plus probables (traduisant très probablement des départs vers l'inactivité). De manière plus surprenante, la présence de jeunes enfants coïncide avec une situation plus favorable pour les hommes : les transitions de l'emploi temporaire vers l'emploi stable sont plus fréquentes, et celles vers le nonemploi moins probables (6).

L'impact du diplôme sur les transitions est également intéressant. Conformément à l'intuition, avoir un diplôme élevé diminue les risques de perte d'emploi stable, et augmente les probabilités de retour à l'emploi quand on en est privé. Le diplôme protège avant tout du risque de non-emploi et influence peu les transitions de l'emploi stable vers l'emploi temporaire. Il n'y a pas d'effet significatif du diplôme sur les probabilités d'accès à un emploi stable pour les salariés hommes en emploi temporaire, alors que pour les femmes, les probabilités d'accéder à un emploi stable augmentent avec le diplôme. Cela peut s'expliquer par le fait que les hommes occupent plus souvent des emplois d'intérim parmi les emplois temporaires, quand les femmes occupent plutôt des CDD. Ces types d'emploi sont parfois utilisés comme période d'essai, et ce profil traduirait en partie le fait que les plus diplômées aient plus de chance de voir convertir leur emploi dans ce cas (7).

Les fonctions de hasard de base mesurent la dépendance de durée : plus précisément, il s'agit de savoir si le fait d'observer telle ou telle transition augmente ou diminue avec le temps passé dans un état. La modélisation d'un hasard constant par morceaux permet d'estimer de manière souple cette dépendance et de mettre en évidence d'éventuels effets non linéaires. De fait, les résultats présentent des profils peu réguliers (cf. graphiques V et VI), ce qui justifie $a$ posteriori cette approche peu paramétrique.

Pour les salariés occupant un emploi temporaire, les intensités de transition vers l'emploi stable sont nettement plus faibles que celles vers le non-emploi. Les transitions vers le non-emploi sont maximales entre trois et six mois. Les transitions vers l'emploi stable sont faibles les trois premiers mois, plus élevées pendant la première année, puis décroissent à nouveau. À l'inverse, les intensités de transition d'emploi temporaire à emploi temporaire sont particulièrement fortes en début de période, mais décroissent plus vite.

Ces profils peuvent s'interpréter par la nature des emplois concernés. Les emplois temporaires sont parfois utilisés comme des périodes d'essai avant une embauche stable. Il est peu probable que celle-ci arrive dans les trois premiers mois. Elle est plus probable avec le temps, mais devient de moins en moins probable à mesure que l'individu persiste en emploi temporaire. Rappelons aussi que, par souci de simplicité, les emplois temporaires regroupent des types de contrats très différents, en particulier en terme d'usages (par les entreprises) et de durées. Des emplois de très courte durée sont donc pris en compte : ils présentent plutôt des risques de rupture élevés, mais avec une forte probabilité de retrouver un tel type d'emploi (ce qui correspond sans doute à des emplois intérimaires). Les emplois en intérim se comptent plutôt en semaines, les emplois en CDD plutôt en mois (8). Il est donc peu surprenant que la probabilité de connaître une perte d'emploi augmente plutôt après trois mois (ce qui peut correspondre à la fin de tels emplois).

Il faut cependant souligner que bien que les cœfficients soient élevés, ils sont estimés avec une faible précision. Il ne faut donc pas surinterpréter ce profil.

La probabilité de sortir du non-emploi pour un emploi temporaire diminue rapidement avec le temps déjà passé hors de l'emploi, et est très faible pour les transitions vers l'emploi stable : ceci confirme que le chômage, comme l'inactivité, seraient des « trappes » dont il s'avère difficile de sortir. Les intensités de transition de l'emploi stable vers un autre emploi sont très faibles (l'ordre de grandeur est plus de cinq fois inférieur à ce qu'on observe pour les transitions venant d'autres états). Les intensités de transition vers le non-emploi sont un peu plus élevées entre trois et six mois, ce qui peut correspondre

6. Ces résultats peuvent évidemment être liés à l'endogénéité possible de cette variable. Dans un modèle traditionnel, des femmes anticipant des difficultés sur le marché du travail pourraient préférer avoir des enfants, tandis que les hommes ne prendraient cette décision que dans des contextes d'amélioration de leur situation professionnelle. Néanmoins, le fait que nous incorporons un terme d'hétérogénéité inobservée dans notre modèle doit théoriquement réduire ce biais.

7. Une autre explication serait que les employeurs hésiteraient plus à embaucher directement une femme en emploi stable.

8. À partir de l'enquête Emploi, nous observons dans Givord et Wilner (2009) que les CDD à temps plein du secteur privé durent en moyenne six mois. 
Graphique V

Taux de hasard prédit (hommes)
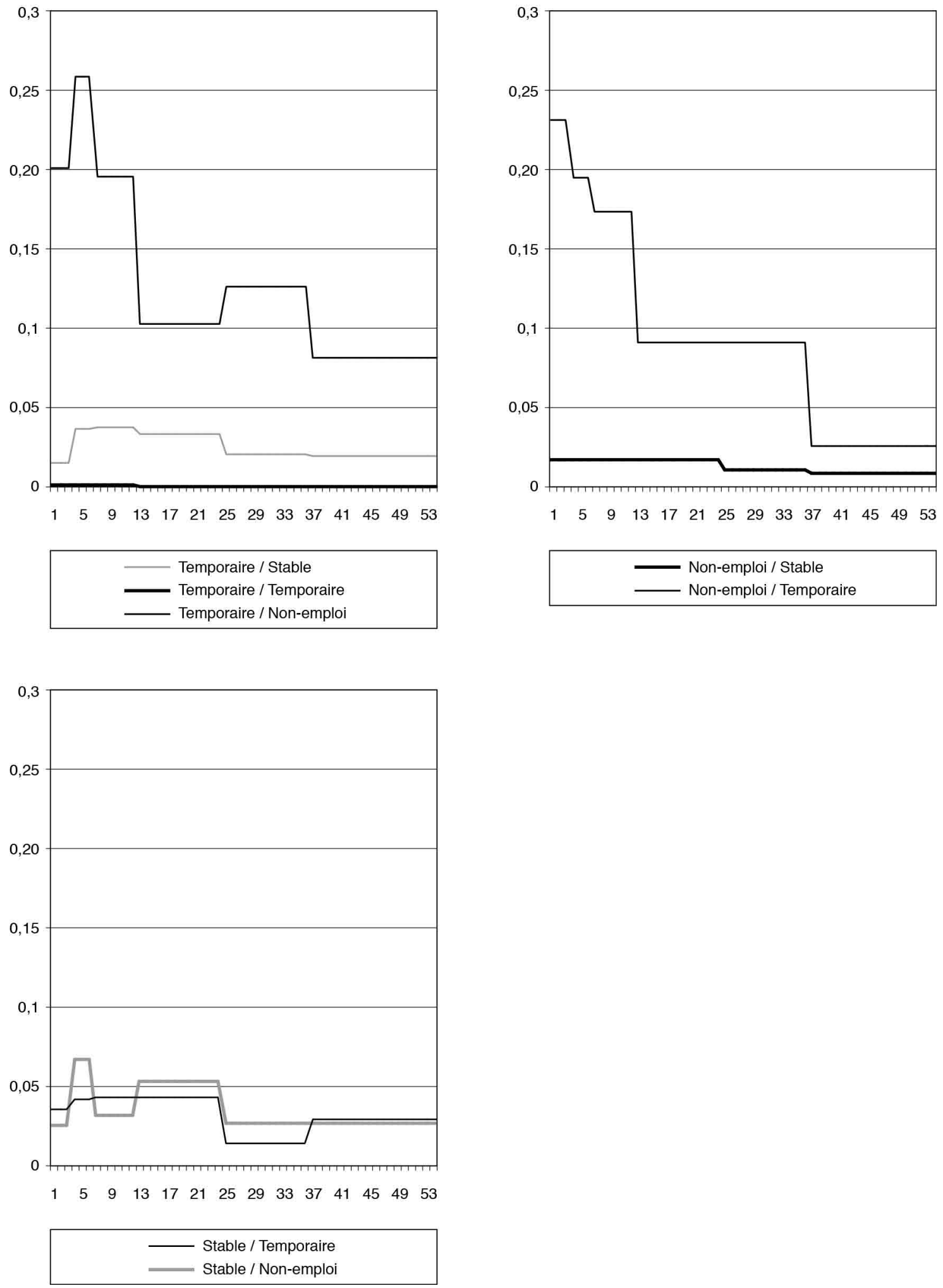

Lecture : taux de hasard mensuel prédit par le modèle (cf. tableau 5).

Champ : hommes de 26 ans sans enfant, ayant un diplôme niveau bac, sans emploi en 1998, ayant occupé un emploi temporaire à l'épisode précédent.

Source : FQP 2003, Insee. 
Graphique VI

Taux de hasard prédit (femmes)
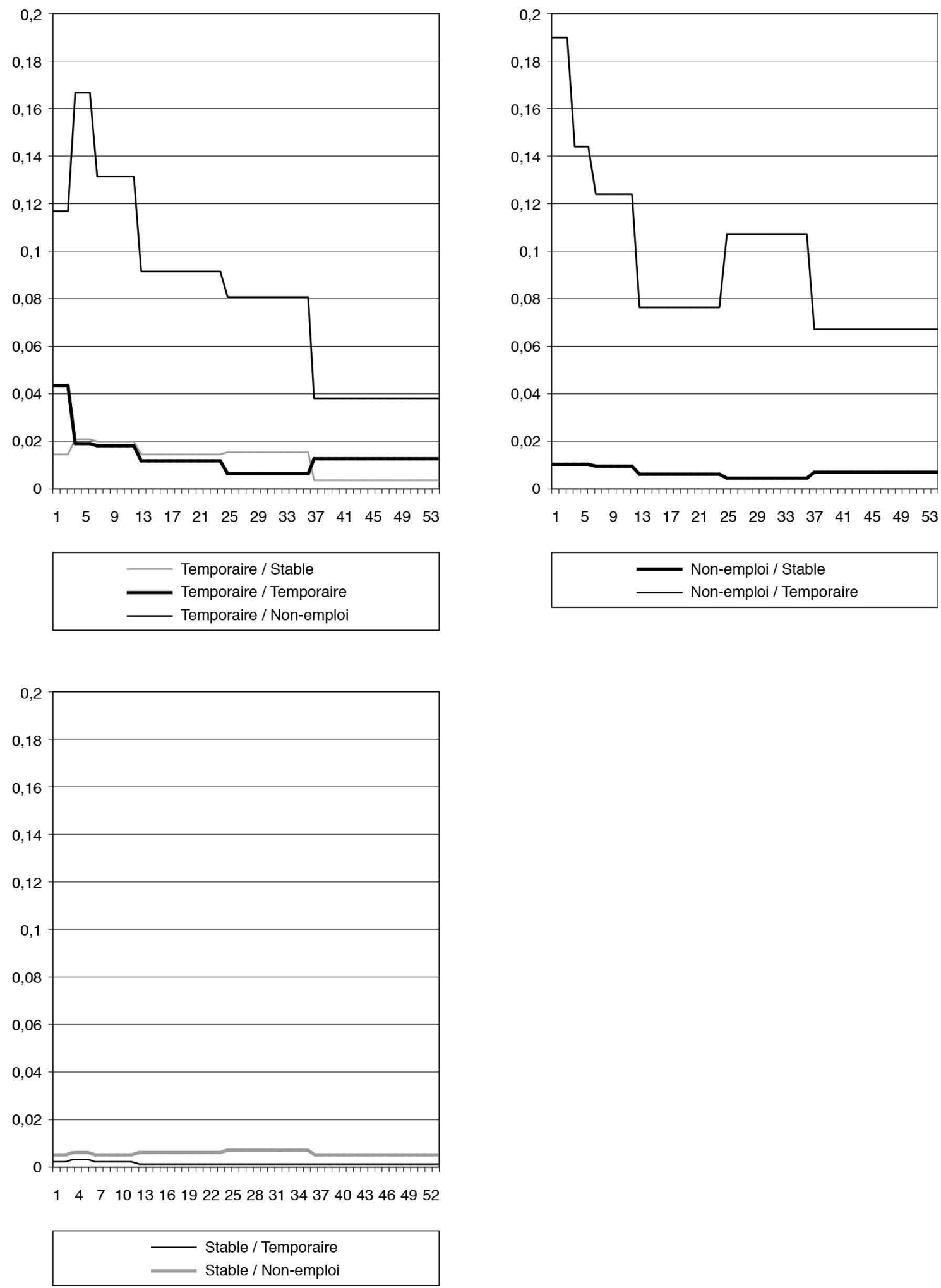

Lecture : taux de hasard mensuel prédit par le modèle (cf. tableau 4).

Champ : femmes de 26 ans sans enfant, ayant un diplôme niveau bac, sans emploi en 1998, ayant occupé un emploi temporaire à l'épisode précédent.

Source: FQP 2003, Insee. 
Tableau 4

Transitions entre emploi temporaire, emploi stable et non-emploi, modèle multi-états multiépisodes (femmes)

\begin{tabular}{|c|c|c|c|c|c|c|c|}
\hline & $\mathrm{St} / \mathrm{Tp}$ & $\mathrm{St} / \mathrm{Ne}$ & $\mathrm{Tp} / \mathrm{St}$ & $\mathrm{Tp} / \mathrm{Tp}$ & $\mathrm{Tp} / \mathrm{Ne}$ & $\mathrm{Ne} / \mathrm{St}$ & $\mathrm{Ne} / \mathrm{Tp}$ \\
\hline \multicolumn{8}{|c|}{ Caractéristiques individuelles } \\
\hline Âge & $\begin{array}{c}-0,17^{\star \star} \\
(0,08)\end{array}$ & $\begin{array}{r}-0,04 \\
(0,03)\end{array}$ & $\begin{array}{r}-0,04 \\
(0,03)\end{array}$ & $\begin{array}{l}-0,01 \\
(0,02)\end{array}$ & $\begin{array}{c}0,02 \\
(0,02)\end{array}$ & $\begin{array}{c}-0,06^{\star \star} \\
(0,02)\end{array}$ & $\begin{array}{l}-0,01 \\
(0,02)\end{array}$ \\
\hline \multicolumn{8}{|l|}{ Diplôme } \\
\hline Aucun & Réf. & Réf. & Réf. & Réf. & Réf. & Réf. & Réf. \\
\hline Inférieur au bac & $\begin{array}{c}-1,10^{\star} \\
(0,63)\end{array}$ & $\begin{array}{c}-0,40^{\star} \\
(0,25)\end{array}$ & $\begin{array}{c}0,28 \\
(0,32)\end{array}$ & $\begin{array}{r}-0,03 \\
(0,21)\end{array}$ & $\begin{array}{r}-0,22 \\
(0,15)\end{array}$ & $\begin{array}{l}0,40^{\star \star} \\
(0,20)\end{array}$ & $\begin{array}{c}0,16 \\
(0,18)\end{array}$ \\
\hline Niveau bac & $\begin{array}{r}-0,60 \\
(0,54)\end{array}$ & $\begin{array}{c}-0,55^{\star \star} \\
(0,24)\end{array}$ & $\begin{array}{l}0,69^{\star *} \\
(0,29)\end{array}$ & $\begin{array}{c}0,04 \\
(0,20)\end{array}$ & $\begin{array}{c}-0,23^{*} \\
(0,15)\end{array}$ & $\begin{array}{l}0,36^{*} \\
(0,20)\end{array}$ & $\begin{array}{l}0,34^{\star *} \\
(0,16)\end{array}$ \\
\hline Supérieur au bac & $\begin{array}{r}-0,27 \\
(0,58)\end{array}$ & $\begin{array}{c}-0,97^{\star \star} \\
(0,27)\end{array}$ & $\begin{array}{l}0,73^{\star *} \\
(0,32)\end{array}$ & $\begin{array}{c}0,15 \\
(0,22)\end{array}$ & $\begin{array}{c}-0,66^{\star \star} \\
(0,17)\end{array}$ & $\begin{array}{l}1,13^{\star *} \\
(0,21)\end{array}$ & $\begin{array}{c}0,06 \\
(0,20)\end{array}$ \\
\hline Enfants $<3$ ans & $\begin{array}{r}-0,23 \\
(0,56)\end{array}$ & $\begin{array}{c}0,09 \\
(0,20)\end{array}$ & $\begin{array}{c}-0,88^{\star \star} \\
(0,33)\end{array}$ & $\begin{array}{l}-0,29 \\
(0,19)\end{array}$ & $\begin{array}{c}0,16 \\
(0,13)\end{array}$ & $\begin{array}{c}-0,40^{\star *} \\
(0,15)\end{array}$ & $\begin{array}{c}-0,46^{\star \star} \\
(0,15)\end{array}$ \\
\hline Nationalité française & $\begin{array}{r}-0,49 \\
(0,76) \\
\end{array}$ & $\begin{array}{c}-0,95^{\star \star} \\
(0,29)\end{array}$ & $\begin{array}{c}0,13 \\
(0,47) \\
\end{array}$ & $\begin{array}{l}0,96^{*} \\
(0,51)\end{array}$ & $\begin{array}{c}0,02 \\
(0,26)\end{array}$ & $\begin{array}{c}0,38 \\
(0,32) \\
\end{array}$ & $\begin{array}{c}0,13 \\
(0,29) \\
\end{array}$ \\
\hline \multicolumn{8}{|c|}{ État occupé à l'épisode précédent } \\
\hline Non emploi & Réf. & Réf. & Réf. & Réf. & Réf. & & \\
\hline Emploi temporaire & $\begin{array}{l}0,64^{*} \\
(0,37)\end{array}$ & $\begin{array}{l}-0,33 \\
(0,22)\end{array}$ & $\begin{array}{l}0,42^{\star \star} \\
(0,19)\end{array}$ & $\begin{array}{l}-0,05 \\
(0,15)\end{array}$ & $\begin{array}{r}-0,09 \\
(0,12)\end{array}$ & $\begin{array}{r}-0,18 \\
(0,14)\end{array}$ & $\begin{array}{c}0,20 \\
(0,13)\end{array}$ \\
\hline Emploi stable & & & $\begin{array}{c}0,52 \\
(0,38) \\
\end{array}$ & $\begin{array}{l}-0,14 \\
(0,37) \\
\end{array}$ & $\begin{array}{r}-0,45 \\
(0,35) \\
\end{array}$ & Réf. & Réf. \\
\hline \multicolumn{8}{|c|}{ Fonction de hasard de base } \\
\hline $0-3$ mois & $\begin{array}{l}-1,27 \\
(2,14)\end{array}$ & $\begin{array}{c}-2,38^{\star \star} \\
(1,00)\end{array}$ & $\begin{array}{c}-4,42^{\star \star} \\
(1,03)\end{array}$ & $\begin{array}{c}-3,83^{\star \star} \\
(0,82)\end{array}$ & $\begin{array}{c}-2,37^{\star \star} \\
(0,53)\end{array}$ & $\begin{array}{c}-3,64^{\star \star} \\
(0,81)\end{array}$ & $\begin{array}{c}-2,07^{\star \star} \\
(0,63)\end{array}$ \\
\hline 3-6 mois & $\begin{array}{l}-1,03 \\
(2,14)\end{array}$ & $\begin{array}{c}-2,28^{\star \star} \\
(1,00)\end{array}$ & $\begin{array}{c}-4,07^{\star *} \\
(1,04)\end{array}$ & $\begin{array}{c}-4,66^{\star \star} \\
(0,83)\end{array}$ & $\begin{array}{c}-2,01^{\star \star} \\
(0,53)\end{array}$ & $\begin{array}{c}-3,60^{\star \star} \\
(0,81)\end{array}$ & $\begin{array}{c}-2,35^{\star \star} \\
(0,63)\end{array}$ \\
\hline 6-12 mois & $\begin{array}{l}-1,60 \\
(2,13)\end{array}$ & $\begin{array}{c}-2,39^{\star *} \\
(0,99)\end{array}$ & $\begin{array}{c}-4,12^{\star \star} \\
(1,05)\end{array}$ & $\begin{array}{c}-4,72^{\star \star} \\
(0,83)\end{array}$ & $\begin{array}{c}-2,25^{\star \star} \\
(0,54)\end{array}$ & $\begin{array}{c}-3,68^{\star *} \\
(0,81)\end{array}$ & $\begin{array}{c}-2,50^{\star *} \\
(0,64)\end{array}$ \\
\hline $12-24$ mois & $\begin{array}{r}-2,02 \\
(2,12)\end{array}$ & $\begin{array}{c}-2,30^{\star \star} \\
(0,98)\end{array}$ & $\begin{array}{c}-4,42^{\star \star} \\
(1,05)\end{array}$ & $\begin{array}{c}-5,14^{\star \star} \\
(0,84)\end{array}$ & $\begin{array}{c}-2,61^{\star *} \\
(0,55)\end{array}$ & $\begin{array}{c}-4,19^{\star *} \\
(0,82)\end{array}$ & $\begin{array}{c}-2,98^{\star *} \\
(0,65)\end{array}$ \\
\hline $24-36$ mois & $\begin{array}{l}-1,71 \\
(2,11)\end{array}$ & $\begin{array}{c}-2,14^{\star \star} \\
(0,97)\end{array}$ & $\begin{array}{c}-4,39^{\star \star} \\
(1,08)\end{array}$ & $\begin{array}{c}-5,75^{\star \star} \\
(0,92)\end{array}$ & $\begin{array}{c}-2,74^{\star \star} \\
(0,57)\end{array}$ & $\begin{array}{c}-4,50^{\star \star} \\
(0,84)\end{array}$ & $\begin{array}{c}-2,64^{\star \star} \\
(0,67)\end{array}$ \\
\hline+36 mois & $\begin{array}{r}-2,42 \\
(2,17) \\
\end{array}$ & $\begin{array}{c}-2,52^{\star \star} \\
(0,98)\end{array}$ & $\begin{array}{c}-5,72^{\star \star} \\
(1,25)\end{array}$ & $\begin{array}{c}-5,09^{\star \star} \\
(0,89)\end{array}$ & $\begin{array}{c}-3,49^{\star \star} \\
(0,65)\end{array}$ & $\begin{array}{c}-4,00^{\star \star} \\
(0,85)\end{array}$ & $\begin{array}{c}-3,11^{\star \star} \\
(0,75)\end{array}$ \\
\hline \multicolumn{8}{|l|}{ Condition initiale } \\
\hline Non-emploi & Réf. & Réf. & Réf. & Réf. & Réf. & Réf. & Réf. \\
\hline Emploi stable & $\begin{array}{l}0,98^{\star *} \\
(0,44)\end{array}$ & $\begin{array}{l}0,45^{\star *} \\
(0,20)\end{array}$ & $\begin{array}{c}0,19 \\
(0,23)\end{array}$ & $\begin{array}{l}-0,03 \\
(0,19)\end{array}$ & $\begin{array}{l}-0,11 \\
(0,14)\end{array}$ & $\begin{array}{l}0,77^{\star \star} \\
(0,17)\end{array}$ & $\begin{array}{l}-0,01 \\
(0,16)\end{array}$ \\
\hline Emploi temporaire & $\begin{array}{c}0,24 \\
(0,42) \\
\end{array}$ & $\begin{array}{c}-0,39^{\star \star} \\
(0,20)\end{array}$ & $\begin{array}{r}-0,19 \\
(0,19) \\
\end{array}$ & $\begin{array}{c}0,03 \\
(0,14)\end{array}$ & $\begin{array}{c}-0,37^{\star *} \\
(0,12) \\
\end{array}$ & $\begin{array}{l}0,34^{\star *} \\
(0,17)\end{array}$ & $\begin{array}{l}0,42^{\star *} \\
(0,13)\end{array}$ \\
\hline \multicolumn{8}{|c|}{ Hétérogénéité inobservée } \\
\hline$v$ & $\begin{array}{c}0,12 \\
(1,12)\end{array}$ & $\begin{array}{c}0,12 \\
(0,56)\end{array}$ & $\begin{array}{c}0,33 \\
(0,53)\end{array}$ & $\begin{array}{c}0,25 \\
(0,31)\end{array}$ & $\begin{array}{c}-0,98^{\star *} \\
(0,17)\end{array}$ & $\begin{array}{l}0,90^{\star *} \\
(0,39)\end{array}$ & $\begin{array}{l}1,11^{\star \star} \\
(0,17)\end{array}$ \\
\hline
\end{tabular}

Lecture : St, Tp et Ne représentent respectivement emploi stable, emploi temporaire et non-emploi ; St/Tp représente par exemple la transition entre emploi stable et emploi temporaire. Voir l'encadré 2 pour la définition des paramètres. Les paramètres correspondant à l'effet des caractéristiques observables figurent dans la première partie du tableau, ceux correspondant à la trajectoire passée dans la seconde. Les hasards de base estimés sont dans la troisième partie du tableau. L'hétérogénéité inobservée est prise en compte par le terme $v$. La probabilité d'être du groupe inobservé pour lequel v est non nul est $\exp (P) /(1+\exp (P))$ avec $P=4,16^{* *}(0,94)$, soit $1,5 \%$.

Les écarts-types sont entre parenthèses. * : significatif au seuil de $5 \% ;{ }^{* *}$ : significatif au seuil de $1 \%$.

Champ : restriction aux 908 femmes sorties de formation initiale entre 1993 et 1997 qui ont au moins un changement d'état entre 1998 et 2003. Nombre d'épisodes utilisés pour l'estimation : 2819.

Source : FQP 2003, Insee. 
Tableau 5

Transitions entre emploi temporaire, emploi stable et non-emploi, modèle multi-états multiépisodes (hommes)

\begin{tabular}{|c|c|c|c|c|c|c|c|}
\hline & $\mathrm{St} / \mathrm{Tp}$ & $\mathrm{St} / \mathrm{Ne}$ & $\mathrm{Tp} / \mathrm{St}$ & $\mathrm{Tp} / \mathrm{Tp}$ & $\mathrm{Tp} / \mathrm{Ne}$ & $\mathrm{Ne} / \mathrm{St}$ & $\mathrm{Ne} / \mathrm{Tp}$ \\
\hline \multicolumn{8}{|c|}{ Caractéristiques individuelles } \\
\hline Âge & $\begin{array}{l}-0,06 \\
(0,09)\end{array}$ & $\begin{array}{r}-0,02 \\
(0,05)\end{array}$ & $\begin{array}{r}-0,01 \\
(0,04)\end{array}$ & $\begin{array}{r}-0,02 \\
(0,03)\end{array}$ & $\begin{array}{c}0,05^{\star \star} \\
(0,02)\end{array}$ & $\begin{array}{r}-0,03 \\
(0,03)\end{array}$ & $\begin{array}{r}-0,04^{\star \star} \\
(0,02)\end{array}$ \\
\hline \multicolumn{8}{|l|}{ Diplôme } \\
\hline Aucun & Réf. & Réf. & Réf. & Réf. & Réf. & Réf. & Réf. \\
\hline Inférieur au bac & $\begin{array}{r}-0,43 \\
(0,49)\end{array}$ & $\begin{array}{r}-0,29 \\
(0,30)\end{array}$ & $\begin{array}{l}0,11 \\
(0,23)\end{array}$ & $\begin{array}{l}0,16 \\
(0,16)\end{array}$ & $\begin{array}{r}-0,08 \\
(0,14)\end{array}$ & $\begin{array}{l}0,39^{\star} \\
(0,21)\end{array}$ & $\begin{array}{c}0,12 \\
(0,14)\end{array}$ \\
\hline Niveau bac & $\begin{array}{r}-0,57 \\
(0,56)\end{array}$ & $\begin{array}{r}-0,74^{\star \star} \\
(0,35)\end{array}$ & $\begin{array}{l}0,13 \\
(0,25)\end{array}$ & $\begin{array}{l}0,07 \\
(0,18)\end{array}$ & $\begin{array}{c}-0,43^{\star \star} \\
(0,16)\end{array}$ & $\begin{array}{c}0,49^{* *} \\
(0,24)\end{array}$ & $\begin{array}{l}0,40^{\star \star} \\
(0,17)\end{array}$ \\
\hline Supérieur au bac & $-0,89$ & $-0,65^{\star}$ & 0,30 & $-0,18$ & $-0,42^{\star \star}$ & $0,96^{\star *}$ & 0,21 \\
\hline & $(0,68)$ & $(0,36)$ & $(0,28)$ & $(0,22)$ & $(0,18)$ & $(0,24)$ & $(0,19)$ \\
\hline Enfants $<3$ ans & $\begin{array}{c}0,48 \\
(0,61)\end{array}$ & $\begin{array}{l}0,21 \\
(0,43)\end{array}$ & $\begin{array}{l}0,44^{*} \\
(0,25)\end{array}$ & $\begin{array}{l}0,11 \\
(0,22)\end{array}$ & $\begin{array}{c}-0,81^{\star *} \\
(0,24)\end{array}$ & $\begin{array}{l}0,30 \\
(0,25)\end{array}$ & $\begin{array}{c}0,04 \\
(0,24)\end{array}$ \\
\hline Nationalité française & $\begin{array}{r}-0,97 \\
(0,67)\end{array}$ & $\begin{array}{r}-0,16 \\
(0,48)\end{array}$ & $\begin{array}{l}0,47 \\
(0,43)\end{array}$ & $\begin{array}{l}0,14 \\
(0,31)\end{array}$ & $\begin{array}{r}-0,16 \\
(0,25)\end{array}$ & $\begin{array}{l}0,07 \\
(0,28)\end{array}$ & $\begin{array}{l}0,61^{\star \star} \\
(0,27)\end{array}$ \\
\hline \multicolumn{8}{|c|}{ État occupé à l'épisode précédent } \\
\hline Non-emploi & Réf. & Réf. & Réf. & Réf. & Réf. & & \\
\hline Emploi temporaire & $\begin{array}{l}0,72^{*} \\
(0,42)\end{array}$ & $\begin{array}{l}0,13 \\
(0,25)\end{array}$ & $\begin{array}{l}0,34^{*} \\
(0,18)\end{array}$ & $\begin{array}{l}0,17 \\
(0,13)\end{array}$ & $\begin{array}{r}-0,20^{*} \\
(0,12)\end{array}$ & $\begin{array}{r}-0,23 \\
(0,16)\end{array}$ & $\begin{array}{r}0,29^{\star *} \\
(0,12)\end{array}$ \\
\hline Emploi stable & & & $\begin{array}{l}0,54 \\
(0,44)\end{array}$ & $\begin{array}{r}-0,16 \\
(0,44)\end{array}$ & $\begin{array}{r}-0,34 \\
(0,44)\end{array}$ & Réf. & Réf. \\
\hline \multicolumn{8}{|c|}{ Fonction de hasard de base } \\
\hline $0-3$ mois & $-0,95$ & $-2,40$ & $-4,88^{\star \star}$ & $-6,46^{\star \star}$ & $-2,11^{\star *}$ & $-3,66^{\star \star}$ & $-1,73^{\star \star}$ \\
\hline & $(2,49)$ & $(2,54)$ & $(1,16)$ & $(0,63)$ & $(0,65)$ & $(1,25)$ & $(0,62)$ \\
\hline $3-6$ mois & $\begin{array}{r}-0,81 \\
(2,46)\end{array}$ & $\begin{array}{l}-1,41 \\
(2,54)\end{array}$ & $\begin{array}{c}-3,98^{\star \star} \\
(1,16)\end{array}$ & $\begin{array}{c}-6,82^{\star \star} \\
(0,63)\end{array}$ & $\begin{array}{c}-1,86^{\star \star} \\
(0,65)\end{array}$ & $\begin{array}{c}-3,66^{\star \star} \\
(1,25)\end{array}$ & $\begin{array}{r}-1,90^{\star \star} \\
(0,63)\end{array}$ \\
\hline $6-12$ mois & $\begin{array}{r}-0,76 \\
(2,43)\end{array}$ & $\begin{array}{l}-2,17 \\
(2,59)\end{array}$ & $\begin{array}{c}-3,97^{\star \star} \\
(1,16)\end{array}$ & $\begin{array}{c}-7,06^{\star \star} \\
(0,64)\end{array}$ & $\begin{array}{c}-2,14^{\star \star} \\
(0,65)\end{array}$ & $\begin{array}{c}-3,71^{\star \star} \\
(1,26)\end{array}$ & $\begin{array}{c}-2,02^{\star \star} \\
(0,63)\end{array}$ \\
\hline $12-24$ mois & $\begin{array}{r}-0,76 \\
(2,38)\end{array}$ & $\begin{array}{r}-1,65 \\
(2,58)\end{array}$ & $\begin{array}{c}-4,08^{\star \star} \\
(1,16)\end{array}$ & $\begin{array}{c}-7,69^{\star \star} \\
(0,65)\end{array}$ & $\begin{array}{c}-2,78^{\star *} \\
(0,66)\end{array}$ & $\begin{array}{c}-3,71^{\star \star} \\
(1,27)\end{array}$ & $\begin{array}{r}-2,67^{\star \star} \\
(0,66)\end{array}$ \\
\hline $24-36$ mois & $\begin{array}{r}-1,90 \\
(2,43)\end{array}$ & $\begin{array}{r}-2,33 \\
(2,57)\end{array}$ & $\begin{array}{c}-4,56^{\star \star} \\
(1,18)\end{array}$ & $\begin{array}{c}-8,16^{\star \star} \\
(0,70)\end{array}$ & $\begin{array}{c}-2,58^{\star \star} \\
(0,66)\end{array}$ & $\begin{array}{c}-4,15^{\star \star} \\
(1,39)\end{array}$ & $\begin{array}{c}-2,67^{\star *} \\
(0,74)\end{array}$ \\
\hline+36 mois & $\begin{array}{r}-1,14 \\
(2,36)\end{array}$ & $\begin{array}{r}-2,33 \\
(2,56)\end{array}$ & $\begin{array}{c}-4,61^{\star \star} \\
(1,22)\end{array}$ & $\begin{array}{c}-7,54^{\star \star} \\
(0,70)\end{array}$ & $\begin{array}{c}-3,02^{\star *} \\
(0,71)\end{array}$ & $\begin{array}{c}-4,40^{\star \star} \\
(1,62)\end{array}$ & $\begin{array}{r}-3,95^{\star \star} \\
(1,18)\end{array}$ \\
\hline \multicolumn{8}{|l|}{ Condition initiale } \\
\hline Non-emploi & Réf. & Réf. & Réf. & Réf. & Réf. & Réf. & Réf. \\
\hline Emploi stable & $\begin{array}{r}-0,45 \\
(0,58)\end{array}$ & $\begin{array}{l}0,45 \\
(0,32)\end{array}$ & $\begin{array}{c}0,61^{\star \star} \\
(0,22)\end{array}$ & $\begin{array}{r}-0,04 \\
(0,19)\end{array}$ & $\begin{array}{c}-0,46^{\star \star} \\
(0,17)\end{array}$ & $\begin{array}{c}0,85^{\star *} \\
(0,23)\end{array}$ & $\begin{array}{r}-0,22 \\
(0,17)\end{array}$ \\
\hline Emploi temporaire & $\begin{array}{r}-0,44 \\
(0,44) \\
\end{array}$ & $\begin{array}{r}-0,00 \\
(0,28) \\
\end{array}$ & $\begin{array}{l}0,01 \\
(0,20)\end{array}$ & $\begin{array}{l}0,10 \\
(0,14)\end{array}$ & $\begin{array}{r}-0,08 \\
(0,12) \\
\end{array}$ & $\begin{array}{l}0,36^{*} \\
(0,22)\end{array}$ & $\begin{array}{c}0,33^{\star \star} \\
(0,13)\end{array}$ \\
\hline \multicolumn{8}{|c|}{ Hétérogénéité inobservée } \\
\hline$v$ & $\begin{array}{c}-2,65^{\star \star} \\
(0,85)\end{array}$ & $\begin{array}{r}-2,24 \\
(1,72)\end{array}$ & $\begin{array}{r}-0,53 \\
(0,66)\end{array}$ & $\begin{array}{c}3,64^{\star *} \\
(0,87)\end{array}$ & $\begin{array}{c}-1,57^{\star \star} \\
(0,26)\end{array}$ & $\begin{array}{c}0,61 \\
(1,03)\end{array}$ & $\begin{array}{r}-0,51 \\
(0,35)\end{array}$ \\
\hline
\end{tabular}

Lecture : St, Tp et Ne représentent respectivement emploi stable, emploi temporaire et non-emploi ; St/Tp représente par exemple la transition entre emploi stable et emploi temporaire. Voir l'encadré 2 pour la définition des paramètres. Les paramètres correspondant à l'effet des caractéristiques observables figurent dans la première partie du tableau, ceux correspondant à la trajectoire passée dans la seconde. Les hasards de base estimés sont dans la troisième partie du tableau. L'hétérogénéité inobservée est prise en compte par le terme v. La probabilité d'être du groupe inobservé pour lequel v est non nul est exp $(P) /(1+\exp (P))$ avec $P=-1,65^{\star *}$, soit $16 \%$.

Les écarts-types sont entre parenthèses. ${ }^{*}$ : significatif au seuil de $5 \%$; ${ }^{* *}$ : significatif au seuil de $1 \%$.

Champ : restriction aux 668 hommes sortis de formation initiale entre 1993 et 1997 qui ont au moins un changement d'état entre 1998 et 2003. Nombre d'épisodes utilisés pour l'estimation : 2269.

Source : FQP 2003, Insee. 
à des périodes d'essai plus ou moins longues. Rappelons que nous ne modélisons pas, par souci de simplicité, les transitions d'emploi stable à emploi stable. Ce résultat ne signifie donc pas forcément que les personnes qui occupent ces emplois demeurent au même poste, mais seulement qu'elles ne transitent pas par le nonemploi ou des emplois temporaires entre deux emplois stables.

Pour tenir compte des phénomènes de dépendances plus complexes dans la trajectoire passée, nous introduisons également des termes mesurant l'état précédent (cf. tableaux 4 et 5). Le message est ambigu. Avoir occupé un emploi temporaire augmente les risques de connaître une transition d'emploi stable vers temporaire, par rapport aux personnes sans emploi durant la période précédente. Avoir occupé un emploi temporaire plutôt que stable à la période précédente augmente également les intensités de transition entre non-emploi et emploi temporaire. Les intensités de transition entre emploi temporaire et emploi stable sont également plus fortes dans ce cas, tandis que les intensités de transitions entre emploi temporaire et non-emploi en sont diminuées. Ces résultats suggèrent que la trajectoire dépend de manière durable des états occupés sur le marché du travail.

L'hétérogénéité inobservée est prise en compte : celle-ci revient à supposer que la population est composée de plusieurs types, ayant des caractéristiques rendant telles ou telles transitions plus probables (cf. encadré 2). Nous avons ici retenu une partition en deux groupes. Pour les femmes, une partition ressort nettement : on obtient ainsi un groupe de près de $16 \%$, qui schématiquement semble avoir un attachement plus fort au marché du travail (intensité de transition du non-emploi vers l'emploi stable plus élevée, moindre transition de l'emploi temporaire vers le non-emploi mais également du non-emploi vers l'emploi temporaire). Pour les hommes, la partition ne ressort pas vraiment, puisque l'on n'isole qu'un sous-groupe de taille très réduite (moins de $2 \%$ de la population). Cette population se caractériserait par des transitions plus fréquentes d'emploi temporaire à emploi temporaire, et moins fréquentes d'emploi temporaire à non-emploi.

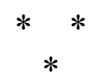

Dans leur ensemble, ces résultats prouvent qu'il est difficile d'apporter des réponses simples à l'analyse de l'impact des contrats temporaires sur l'insertion. Les estimations mettent en évidence l'influence importante des caractéristiques individuelles sur les transitions. Elles montrent également que le fait de passer par tel ou tel état du marché du travail peut avoir un effet à long terme sur la trajectoire professionnelle. Le fait de passer par un emploi temporaire en début de carrière peut donc avoir une incidence sur le plus long terme. Néanmoins, si la complexité du modèle utilisé ici permet de rendre compte de celle des mécanismes à l'œuvre, elle constitue également sa limite. Les conditions d'identification de l'ensemble des paramètres correspondant à la trajectoire passée ne sont pas toujours clairement établies (pour une discussion voir Baker et Melino, 2000). Compte tenu de la taille réduite de l'échantillon utilisé ici, il nous semble cependant difficile d'aller plus loin. Une extension intéressante serait d'utiliser les enquêtes Génération du Cereq. 


\section{BIBLIOGRAPHIE}

Abbring J.H. et van den Berg G.J. (2003), « The Identifiability of the Mixed Proportional Hazards Competing Risks Model », Journal of the Royal Statistical Society : Series B (Statistical Methodology), vol. 65, $\mathrm{n}^{\circ} 3$, pp. 701-710.

Autor D. et Houseman N. (2005), « Do Temporary Help Jobs Improve Labor Market Outcomes for Low-Skilled Workers ? Evidence from Random Assignments », NBER Working Paper, n 11743.

Baker M. et Melino A. (2000), « Duration Dependence and Nonparametric Heterogeneity : A Monte Carlo Study », Journal of Econometrics, vol. $96, \mathrm{n}^{\circ} 2$, pp. 357-393.

Beffy M., Coudin E. et Rathelot R. (2008), «Who is Confronted to Insecure Labor Market Histories? Some Evidence Based on French Labor Market Transitions », Document de Travail Insee, $\mathrm{n}^{\circ} \mathrm{G} 2008 / 10$.

Bonnal L., Fougère D. et Sérandon A. (1997), « Evaluating the Impact of French Employment Policies on Individual Labour Market Histories », Review of Economic Studies n ${ }^{\circ}$ 64, pp. 683-713.

Booth A.L., Francesconi M. et Frank J. (2002), « Temporary Jobs : Stepping Stones or Dead Ends ? », Economic Journal, vol. 112(480), pp. 189-213.

Borjas G.J. et Heckman J.J. (1980), « Does Unemployment Cause Future Unemployment ? Definitions, Questions, and Answers from a Continuous Time Model of Heterogeneity and State Dependence », Economica, vol. 47, pp. 247-283.

Calavrezo O. (2008), « The Effects of Fixed-Term Employment on the Integration of School-leavers on the Labour Market : Evidence from France », International Journal for Quality Research, vol. 1, $\mathrm{n}^{\circ} 4$.

Cancé R. et Fréchou R. (2003), « Les contrats courts : source d'instabilités mais aussi tremplin vers l'emploi permanent », Premières Informations et Premières Synthèses, $\mathrm{n}^{\circ}$ 14.1, Dares.

Chauvin S. (2010), Les Agences de la précarité : journaliers à Chicago, Le Seuil, Paris.
Cockx B. et Picchio M. (2009), « Are Short-Lived Jobs Stepping Stones to Long-Lasting Jobs ? », IZA Discussion Papers, ${ }^{\circ} 4007$.

Doiron D. et Gorgens T. (2008), « State Dependence in Youth Labor Market Experiences, and the Evaluation of Policy Interventions ", Journal of Econometrics, vol. 145, n ${ }^{\circ} 1-2$, pp. 81-97.

Fondeur Y. et Minni C. (2006), « L'accès des jeunes à l'emploi », Données sociales - La société française - Édition 2006.

Gagliarducci S. (2005), " The Dynamics of Repeated Temporary Jobs ", Labour Economics, vol. 12(4), pp. 429-448.

Garcia Pérez J.I. et Muñoz-Bullon F. (2007), « Transitions into Permanent Employment in Spain : An Empirical Analysis for Young Workers ", Working Paper 07-38, Business Economics Series 08, Universidad Carlos III, Departamento de Economia de la Empresa.

Goebel C. Et Verhofstadt E. (2008), « Is Temporary Employment a Stepping Stone for Unemployed School Leavers ? », ZEW - Centre for European Economic Research Discussion Paper, $\mathrm{n}^{\circ}$ 08-093.

Givord P. (2006), « Formes particulières d'emploi et insertion des jeunes », Économie et Statistique, $\mathrm{n}^{\circ}$ 388-389, pp. 145-169.

Givord P. et Wilner L. (2009), " Fixed-Term Contracts, Incentives and Effort », Document de Travail Crest, $\mathrm{n}^{\circ}$ 2009-15.

Havet N. (2006), « L'insertion professionnelle des jeunes et mesures publiques : des trajectoires différenciées entre hommes et femmes », Annales d'Économie et Statistiques, ${ }^{\circ} 81$, pp. 225-250.

Heckman, J.J. et Singer, B. (1984), « A Method for Minimizing the Impact of Distributional Assumptions in Econometric Models for Duration Data », Econometrica, vol. 52, n 2, pp. 271-320.

Horny G. et Picchio M. (2009), « Identification of Lagged Duration Dependence in Multiple Spells Competing Risks Models », Discussion 
Papers (Ires - Institut de Recherches Économiques et Sociales), n 2009001.

Ichino A., Mealli F. et Nannicini T. (2008), « From Temporary Help Jobs to Permanent Employment: What Can We Learn from Matching Estimators and their Sensitivity ? ", Journal of Applied Econometrics, John Wiley et Sons, Ltd., vol. $23, \mathrm{n}^{\circ} 3$, pp. 305-327.

Lancaster T. (1990), The Econometric Analysis of Transition Data, Cambridge University Press, New-York, USA.

Lollivier S. (2000), « Récurrence du chômage dans l'insertion des jeunes : Des trajectoires hétérogènes », Économie et Statistique, n 334, pp. 49-63.

Lopez A. (2004), « Les modes de stabilisation en emploi en début de vie active ", Économie et Statistique $\mathrm{n}^{\circ}$ 378-379, pp. 105-128.

Lutinier B. (2007), « Les mouvements de maind'œuvre au troisième trimestre 2006 », Premières synthèses $\mathrm{n}^{\circ} 38.4$, Dares.

Magnac T. (2000), « Subsidised Training and Youth Employment : Distinguishing Unobserved Heterogeneity from State Dependence in Labour Market Histories ", The Economic Journal, vol. 110 , pp. 805-837.
McGinnity F., Mertens A. et Gundert S. (2005), «A Bad Start ? Fixed-Term Contracts and the Transition from Education to Work in West Germany », European Sociological Review, vol. $21, \mathrm{n}^{\circ} 4$, pp. $359-374$.

Paugam S. (2000), Le salarié de la précarité. Les nouvelles formes de l'intégration professionnelle, Presses Universitaires de France, coll. « Le lien Social », Série « Documents d'enquête ».

van den Berg G.J. (2001), « Duration Models : Specification, Identification and Multiple Durations », Handbook of Econometrics, vol. 5, Heckman et Leamer (eds).

van den Berg G.J. et van der Klaauw B. (2001), "Combining Micro and Macro Unemployment Duration Data ", Journal of Econometrics, vol. 102, n ${ }^{\circ} 2$, pp. 271-309.

Wooldridge J. (2005), « Simple Solutions to the Initial Conditions Problem in Dynamics, Nonlinear Panel Data Models with Unobserved Heterogeneity », Journal of Applied Econometrics, vol. $20, \mathrm{n}^{\circ} 1$, pp. 39-54.

Zijl M., van den Berg G.J. et Heyma A. (2004), «Stepping Stones for the Unemployed : the Effect of Temporary Jobs on the Duration Until Regular Work », IZA Discussion Paper n 1241. 


\section{État des lieux des régions françaises...}

Collection Insee Références

\section{La France
et ses régions \\ La France
et ses régions}

홇

Édition 2010

$>$ Six dossiers dont

- les régions et les départements face à la pauvreté

- I'attractivité des territoires

- les perspectives de la démographie médicale

- etc

$>$ Et aussi, le positionnement

des régions dans le cadre européen

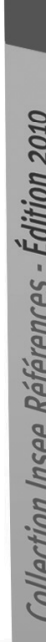

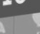

En vente dans les librairies, à I'Insee et sur www.insee.fr 